\title{
Influence of aspect ratio on vortex formation in X-junctions: Direct numerical simulations and eigenmode decomposition
}

Cite as: Phys. Fluids 32, 124105 (2020); https://doi.org/10.1063/5.0026829

Submitted: 25 August 2020 . Accepted: 12 November 2020 . Published Online: 04 December 2020

P. G. Correa, J. M. Gomba, (D) J. R. Mac Intyre, (D) S. Ubal, C. A. Perazzo, (D) J. P. Hulin, and (D) H. Auradou

\section{COLLECTIONS}

EP This paper was selected as an Editor's Pick
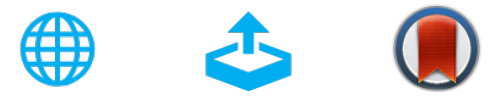

\section{ARTICLES YOU MAY BE INTERESTED IN}

Modeling surfactant-laden droplet dynamics by lattice Boltzmann method

Physics of Fluids 32, 122105 (2020); https://doi.org/10.1063/5.0028554

Extension at the downstream end of turbulent band in channel flow

Physics of Fluids 32, 121703 (2020); https://doi.org/10.1063/5.0032272

Rough-wall turbulence in minimal flow units with rod-roughened walls

Physics of Fluids 32, 115120 (2020); https://doi.org/10.1063/5.0028015

\section{Physics of Fluids GALLERY OF GOVERS}




\title{
Influence of aspect ratio on vortex formation in X-junctions: Direct numerical simulations and eigenmode decomposition 라
}

\author{
Cite as: Phys. Fluids 32, 124105 (2020); doi: 10.1063/5.0026829 \\ Submitted: 25 August 2020 - Accepted: 12 November 2020 • \\ Published Online: 4 December 2020
}

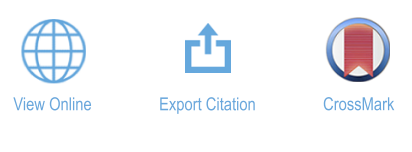

\author{
P. G. Correa, ${ }^{1, a)}$ J. M. Gomba, ${ }^{1, b)}$ J. R. Mac Intyre, ${ }^{2}$ (D) S. Ubal, ${ }^{3}$ (D) C. A. Perazzo, ${ }^{4}$ J. P. Hulin, \\ and $\mathrm{H}$. Auradou ${ }^{5}$
}

\begin{abstract}
AFFILIATIONS
${ }^{2}$ Department of Applied Physics, School of Science, Aalto University, P.O. Box 11100, 00076 Aalto, Finland

${ }^{3}$ Instituto de Investigación y Desarrollo en Bioingeniería y Bioinformática, UNER-CONICET, Ruta Prov. 11 KM 10,

3101 Oro Verde, Argentina

4IMeTTyB, Universidad Favaloro-CONICET, Solís 453, C1078AAI Buenos Aires, Argentina

${ }^{5}$ Université Paris-Saclay, CNRS, FAST, 91405 Orsay, France

a) Author to whom correspondence should be addressed: pcorrea@exa.unicen.edu.ar

b) Electronic addresses: jgomba@exa.unicen.edu.ar and jgomba@engineering.ucsb.edu

${ }^{c}$ Electronic mail: hulin@fast.u-psud.fr
\end{abstract}

${ }^{1}$ Instituto de Física Arroyo Seco IFAS (UNCPBA) and CIFICEN (UNCPBA-CICPBA-CONICET), Pinto 399, 7000 Tandil, Argentina

\begin{abstract}
We study numerically the appearance and number of axial vortices in the outlets of X-shaped junctions of two perpendicular channels of rectangular sections with facing inlets. We explore the effect of the aspect ratio of the cross section, $A R$, on the number of vortices created at the center of the junction. Direct numerical simulations (DNSs) performed for different values of the Reynolds number Re and $A R$ demonstrate that vortices with their axis parallel to the outlets, referred to as axial vortices, appear above critical Reynolds numbers $R e_{c}$. As $A R$ increases from 1 to 11 , the number of vortices observed increases from 1 to 4 , independently of $R e$. For $A R=1$, the single axial vortex induces an interpenetration of the inlet fluids in the whole section; instead, for larger $A R$ 's for which more vortices appear, the two inlet fluids remain largely segregated in bands, except close to the vortices. The linear stability analysis demonstrates that only one leading eigenmode is unstable for a given set of values of $A R$ and $R e$. This mode provides a simplified model of the flow field, reproducing its key features such as the number of vortices and their distance. Its determination with this method requires a much smaller computational load than the DNS. This approach is shown to allow one to determine quickly and precisely the critical Reynolds number $R e_{c}$ and the sensitivity function $S$, which characterizes the influence of variations of the base flow on the unstable one.
\end{abstract}

Published under license by AIP Publishing. https://doi.org/10.1063/5.0026829

\section{INTRODUCTION}

One of the challenges of the lab-on-a-chip technology is to develop a microreactor and analytical equipment that operates at the scale of a few micrometers and can be used to mix, ${ }^{1,2}$ separate, ${ }^{3-5}$ trap, ${ }^{6}$ and transport chemicals or small particles. ${ }^{7}$ To allow all those operations, a perfect control of the fluids and of their flow is required. Knowing and understanding how vortices are created is, for instance, crucial to combine fluids and to ensure a good mixing between them in order to enhance mass or heat transfer. ${ }^{8}$ However, due to the small dimensions of those systems and because of the viscosity of the fluids, vortices cannot be obtained by making the fluid turbulent. Yet, even in relatively viscous flows and in geometries of small size, vortices can be created by carving cavities ${ }^{9}$ or by deviating and bending the flow, thereby playing with the channel's geometry. ${ }^{10,11}$ This can be achieved by injecting fluids in $\mathrm{X}$-junctions and $\mathrm{T}$-junctions in opposing channels: this provides hope for the development of passive mixers, ${ }^{12}$ using only static parts to promote mixing without the need of any external energy supply or movable elements. ${ }^{10}$ Another important practical issue is the active control of 
the flow within such junctions and, particularly, of the development of instabilities. ${ }^{13}$

Originally, X-junctions were developed because their geometry allows for the generation of a stagnation point away from channel walls. In the vicinity of this particular point, the flow is purely planar, elongational, and free of shear and vorticity along the symmetry axes. ${ }^{14}$ These properties have been put at work in many areas of research, including studies of polymer macromolecules dynamics, such as DNA ${ }^{15,16}$ or fluid rheology, ${ }^{17-21}$ and for imposing controlled deformations to cells, vesicles, or droplets. ${ }^{22}$

The flow field is quite simple at low velocity, but increasing it leads to a more complex vorticity field in the intersection and in the outlets. For a Newtonian fluid of constant viscosity $v$, the onset of the different regimes is determined by the Reynolds number $R e=\widetilde{U} \widetilde{W} / v$, where $\widetilde{U}$ is a characteristic velocity and $\widetilde{W}$ is the dimensional width of the channels. For an X-junction with channels crossing at an angle $\alpha=90^{\circ}$, the flow along the outlet channels at low $R e$ 's is symmetric with respect to the plane $y=0$ [see Figs. 1(b) and $1(\mathrm{c})$ ]: the two injected fluids remain segregated. At higher Re's, an axial vortex appears at the intersection of the channels and extends toward each outlet. In channels with a square cross section, ${ }^{8,20,23}$ the transition between the two regimes occurs for $R e_{c} \sim 40$, while for circular channels, ${ }^{24}$ it is slightly higher with $R e_{c} \sim 48$.

In these square cross section junctions, the redirection of the flow around the corners at the junction of the streams leads to the formation of small Dean vortices at the intersection of the channels, ${ }^{25,26}$ even for $R e<R e_{c}$. This secondary flow consists of a double pair of counter-rotating vortices positioned symmetrically at the four corners of the outlet channels, but out of the $z$-axis (see Fig. 1 in Ref. 27). Both fluids remain segregated by the plane $y=0$ despite the presence of these small structures. The intensity of these vortices increases with the flow rate, and at the critical Reynolds number $R e_{c}$, two opposite vortices, out of the four, intensify and the symmetry is broken. The latter vortices finally merge into a single, steady, streamwise, vortex centered on the $z$-axis. This vortex was shown recently to be strong enough to deform the membrane of living cells that in turn become porous to nanomaterials, ${ }^{28}$ which opens the possibility to use such flows for hydroporation. The creation of "hot spots" by the vortices where chemical reactions are enhanced was also found to be a possible method to study chemical reactions. ${ }^{29-32}$ If the velocity is further increased, the flow becomes unsteady at $R e \sim 100$ and periodic oscillations are observed. ${ }^{26}$

The dynamics of the destabilization and merging of the Dean vortices was studied experimentally using time-resolved flow velocimetry by Burshtein et al. ${ }^{27}$ They also investigated the influence of the aspect ratio $A R=\widetilde{H} / \widetilde{W}$, where $\widetilde{W}$ and $\widetilde{H}$ are, respectively, the width and height of the channels, on the dynamics of the formation of the central vortex. They confirmed that the geometry influences the nature of the transition. For wide channels corresponding to $A R<0.5$, the flow recovers its symmetry at similar $R e_{c}$ values for both decreasing or increasing inlet velocity variation ramps. For $A R \geq 0.5$, the transition changes and becomes subcritical. As a result, when the flow velocity decreases, the flow configuration reverts from a single vortex centered in the outlet channel to a Dean-like one with symmetric vortices at a Reynolds number $R e_{c}^{*}$, lower than $R e_{c}$. This confirmed previous results obtained numerically by Haward et al.: $:^{23}$ these authors have shown that the symmetry-breaking flow bifurcation that is supercritical (non-symmetric) for wide channels becomes subcritical (pitchfork bifurcation) for deep ones. The former studies demonstrate, therefore, that the geometry of the channel section influences the critical Reynolds number for the transition between the different regimes. Similar observations were made in $\mathrm{Y}$-junctions or $\mathrm{T}$-junctions ${ }^{33}$ and in $\mathrm{X}$-junctions with varying crossing angles. ${ }^{24}$

All these studies dealt with the dynamics of vortex formation and of the steady "engulfment" regime at relatively low values of $A R$ $(0.4<A R<3.8)$. Moreover, in this range, there is only one axial vortex inside the flow section. Higher aspect ratios $(A R \gtrsim 4)$ are, however, also of interest, for instance, for rheology, because high $A R$ 's ensure that the strain rate is approximately uniform throughout the height, which makes rheological measurements easier. To our knowledge, larger aspect ratios were only used in the experiments of Kalashnikov and Tsiklauri. ${ }^{34}$ For very large values $A R$ $=262$ and $A R=32$, these authors observed a periodic array of vortices stacked over the full height of the channels. When increasing the flow, this structure appeared above respective critical Reynolds numbers $R e_{c}=55$ and $R e_{c} \sim 43$. The transition is, again, subcritical so that, when the flow is lowered, the vortices disappear for $R e_{c}$ $=38.5$ and $R e_{c} \sim 30$, respectively. Qualitative visualizations suggested that such devices may mix large quantities of fluids at a relatively low Reynolds number.

Recently, the so-called "structural sensitivity" formalism has been developed to predict the effect of perturbations brought to a flow in localized regions: ${ }^{35,36}$ it allows detecting the locations where an external actuation either triggers an instability or delays it. Giannetti and Luchini ${ }^{35}$ and Marquet et al. ${ }^{37}$ applied this approach to a two-dimensional analysis of the wake generation downstream of a stationary cylinder. Although the magnitude of linear instability modes increases continuously with the distance, the region where a feedback force produces the largest change in the leading eigenvalues (i.e., where the structural sensitivity is largest) is close to the obstacle and is the best location to control the wake generation. Using the same approach, Lashgari et al..$^{38}$ studied the flow instabilities in an X-junction (three inlets, one outlet), for which the base flow is two-dimensional and the perturbations are decomposed into biglobal modes. ${ }^{39}$ In this case, the sensitivity is highest at the edge of recirculation bubbles at the corners of the junction. Chen et al. ${ }^{36}$ performed a global sensitivity analysis to study flow through a T-shaped pipe bifurcation. They observed recirculation zones resembling the traditional bubble-type breakdown. These regions are highly sensitive to localized feedback forces, and in contrast to observations on three inlet X-junctions, ${ }^{38}$ the flow separation at the corners of the $\mathrm{T}$-junction does not display a clear-cut sensitivity.

The present paper is devoted to the numerical study of the transitions between the different regimes discussed above for flow in Xjunctions of channels of rectangular cross sections. More specifically, we report direct numerical simulation (DNS) for $A R$ values between 1 and 11 corresponding to the appearance of $1-4$ vortices at the junction intersection and along the height ( $z$-axis); note that these computations become more demanding as $A R$ increases. For each value of $A R$, the influence of the Reynolds number on the structure of the flow is studied at Reynolds numbers below 100 corresponding to stationary flow regimes and the possible subcritical nature of the instability leading to the appearance of the vortices is investigated. We analyze, in particular, whether the number of vortices in 
(a)

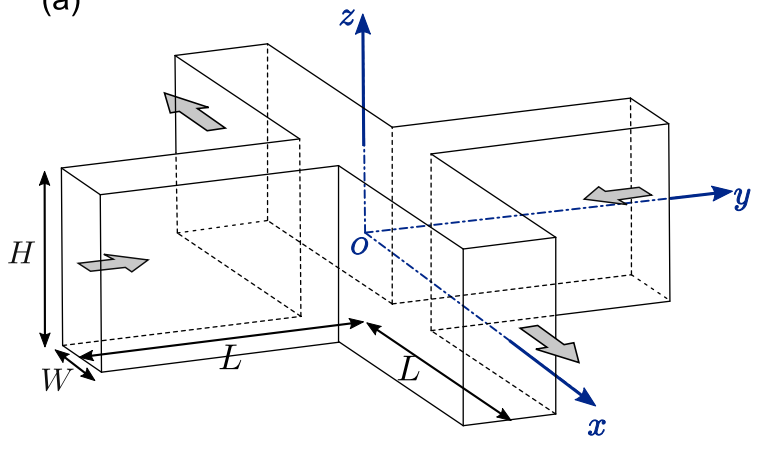

(b)

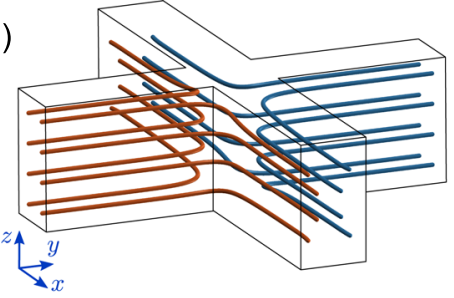

(c)

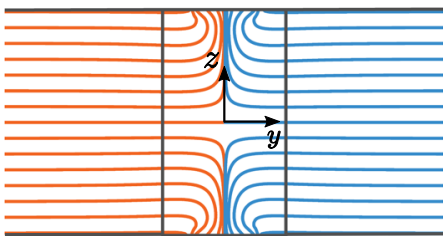

FIG. 1. (a) X-junction of channels of rectangular cross sections with the normalized width and height $W$ and $H$, respectively. The inlets are perpendicular to the outlets. (b) Perspective view of streamlines corresponding to the segregated base flow $\left\{\boldsymbol{u}_{0}, p_{0}\right\}$ discussed in Sec. II A. Streamlines corresponding to the fluid entering each inlet are labeled with a different color. (c) Streamlines of the base flow $\left\{u_{0}, p_{0}\right\}$ in the plane $x=0$. The streamlines shown in graphs (b) and (c) correspond to $R e=22$ and $A R=1.8$. the height only depends on $A R$ or whether $R e$ also has an influence. A three-dimensional global stability analysis has then been performed to model the instability leading to the formation of the vortices: we shall compare its predictions for the critical values of $A R$ and $R e$ and the number and spacing of vortices to those of the DNS. The sensitivity of the flow to the application of feedback forces is finally considered, which may be of interest for flow control applications.

\section{PROBLEM STATEMENT AND FORMULATION}

We consider the flow of two facing incompressible Newtonian liquids within an $\mathrm{X}$-junction with an angle $90^{\circ}$ between the inlet and outlet channels. The device consists of two perpendicular channels of length $2 L$ and identical rectangular cross sections, with the inlets aligned with the $y$-axis and the outlets parallel to the $x$-axis, as shown in Fig. 1(a). The dimensional width and height of the channels are $\widetilde{W}$ and $\widetilde{H}$, respectively, $A R=\widetilde{H} / \widetilde{W}$ being the aspect ratio. The origin $O$ of the coordinate system is located at the center of symmetry of the junction. In the following, all lengths are normalized by the width $\widetilde{W}$ and the velocities by the mean velocity at the entrances, $\widetilde{U}_{i n}$. With these scales, the normalized height and width of the channels are, respectively, $H(=A R)$ and $W(=1)$.

The flow within the junction is governed by the time-dependent three-dimensional incompressible Navier-Stokes equations,

$$
\begin{gathered}
\frac{\partial \boldsymbol{u}}{\partial t}+(\boldsymbol{u} \cdot \nabla) \boldsymbol{u}=-\nabla p+\frac{1}{R e} \nabla^{2} \boldsymbol{u}, \\
\nabla \cdot \boldsymbol{u}=0,
\end{gathered}
$$

where $\boldsymbol{u} \equiv(u, v, w)$ and $p$ are the normalized velocity and pressure, respectively. The Reynolds number is $R e=\widetilde{U}_{i n} \widetilde{W} / v$, where $v$ is the viscosity of the liquid. We impose the same parabolic inflow conditions at both inlets (Poiseuille solution for rectangular pipes), a stress-free outflow at the outlets and $\boldsymbol{u}=0$ at the walls.

\section{A. Linear stability analysis}

To perform a standard global linear stability analysis (LSA), ${ }^{35,37,40}$ the variables in Eq. (1) are written as the sum of a steady base flow $\left\{\boldsymbol{u}_{0}, p_{0}\right\}$ and an unsteady small perturbation $\left\{\boldsymbol{u}^{\prime}, p^{\prime}\right\}$. The base state shares the same initial and boundary conditions as $\{\boldsymbol{u}, p\}$.
In our case, it is a symmetrical solution of Eq. (1) in which the liquid coming from each inlet splits equally between both outlets, with the streamlines completely segregated by the plane $y=0$, as shown in Figs. 1(b) and 1(c). 23,24,41

The perturbations are decomposed into global modes, i.e., $\left\{\boldsymbol{u}^{\prime}\right.$, $\left.p^{\prime}\right\}=\{\hat{\boldsymbol{u}}, \hat{p}\}(x, y, z) \exp (\lambda t)$, where $\lambda=\sigma+i \omega$ is a complex eigenvalue. The real part $\sigma$ is the growth rate, and the imaginary part $\omega$ is the oscillation angular frequency of the perturbation. If the growth rate is positive for, at least, one eigenvalue, the flow is linearly unstable; otherwise, the perturbation decays to zero. This linearization of the flow around the base flow and the subsequent eigenmode decomposition result in the following direct eigenvalue equation for the perturbations: ${ }^{35,36}$

$$
\begin{gathered}
\lambda \hat{\boldsymbol{u}}+\left(\boldsymbol{u}_{0} \cdot \nabla\right) \hat{\boldsymbol{u}}+(\hat{\boldsymbol{u}} \cdot \nabla) \boldsymbol{u}_{0}=\frac{1}{R e} \nabla^{2} \hat{\boldsymbol{u}}-\nabla \hat{p}, \\
\nabla \cdot \hat{\boldsymbol{u}}=0 .
\end{gathered}
$$

Since $\{\boldsymbol{u}, p\}$ and $\left\{\boldsymbol{u}_{0}, p_{0}\right\}$ share the same boundary conditions, the perturbations satisfy homogeneous conditions at all boundaries. The components of each velocity field are $\boldsymbol{u}_{0}:=\left(u_{0}, v_{0}, w_{0}\right)$ and $\hat{\boldsymbol{u}}$ $:=(\hat{u}, \hat{v}, \hat{w})$.

\section{B. Adjoint problem and structural sensitivity}

The adjoint of a linear operator is a useful concept in functional analysis that has been widely applied to problems in turbulence control, receptivity, and transition, and it has recently been used for the analysis of flow within micro-junctions. ${ }^{36,40}$ Following Chomaz, ${ }^{42}$ and in order to evaluate the sensitivity of the solutions of Eq. (1), we apply some well known concepts related to the adjoint problem. In the next lines, we summarize the main equations that we need to solve in order to compute the sensitivity function. A more detailed derivation of this theory can be found in Refs. 35, 36, 42, and 43 . Hill ${ }^{43}$ and Giannetti and Luchini ${ }^{35}$ developed the theory of structural analysis and showed that the adjoint fields $\left\{\boldsymbol{u}^{+}, p^{+}\right\}$ $=\left\{\hat{\boldsymbol{u}}^{+}, \hat{p}^{+}\right\}(x, y, z) \exp (-\lambda t)$ associated with the global mode $\{\hat{\boldsymbol{u}}, \hat{p}, \lambda\}$ satisfy the eigenvalue problem,

$$
-\lambda^{*} \hat{\boldsymbol{u}}^{+}-\left(\nabla \boldsymbol{u}_{0}\right) \cdot \hat{\boldsymbol{u}}^{+}+\boldsymbol{u}_{0} \cdot \nabla \hat{\boldsymbol{u}}^{+}=\nabla \hat{p}^{+}-\frac{1}{R e} \nabla^{2} \hat{\boldsymbol{u}}^{+},
$$




$$
\nabla \cdot \hat{\boldsymbol{u}}^{+}=0
$$

Here, we use the notation $((\nabla \boldsymbol{a}) \cdot \boldsymbol{b})_{i}:=\sum_{j} b_{j} \partial a_{j} / \partial x_{i} .{ }^{36}$ The boundary conditions for the adjoint modes are $\hat{p}^{+} \mathbf{n}=\left(\boldsymbol{u}_{0} \cdot \mathbf{n}\right) \hat{\boldsymbol{u}}^{+}$ $+\operatorname{Re}^{-1}\left(\mathbf{n} \cdot \nabla \hat{\boldsymbol{u}}^{+}\right)$at the outlets ${ }^{37,40}$ and $\hat{\boldsymbol{u}}^{+}=0$ for the rest of the boundaries.

Solving Eqs. (2) and (3) allows one to detect the core region of the instability (for some geometries, the region is named wavemaker $^{38,42,44,45}$ ), i.e., the spots where a local feedback force results in the largest drift of the most "dangerous" eigenvalue. ${ }^{35,36}$ This force could be, for example, the result of the action of an actuator that reacts to the local velocity of the flow at the point where the actuator is operating. Giannetti and Luchini found that the maximum change in the dominant eigenvalue is induced at the location of the greatest overlap of the direct and adjoint modes and defined the structural sensitivity function as ${ }^{35,45}$

$$
S(x, y, z)=\frac{\|\hat{\boldsymbol{u}}\|\left\|\hat{\boldsymbol{u}}^{+}\right\|}{\left\langle\hat{\boldsymbol{u}}, \hat{\boldsymbol{u}}^{+}\right\rangle},
$$

where $\langle\boldsymbol{a}, \boldsymbol{b}\rangle \equiv \int_{V} \boldsymbol{a}^{*} \cdot \boldsymbol{b} d V$, the asterisk denoting the conjugate of a complex quantity. ${ }^{37,40}$ The regions of the flow where $S$ is large are the sites where a feedback force will produce the strongest disturbance on the flow, and it identifies the region where the triggering of the instability occurs. ${ }^{42}$ Note that the location of large values of the direct eigenfunction does not necessarily play a special role in determining the spectrum of a stability equation unless the adjoint eigenfunction is also large at the same spot. This fact is also helpful for the numerical simulations because it gives a criterion for detecting the region where the mesh should be denser in order to capture accurately the global modes.

\section{Numerical implementation and validation}

Equations (1)-(3) were discretized and solved numerically by a finite element method. Polynomial shape functions P2 and P1 were used for the discretization of the velocity and pressure, respectively. The time-dependent Navier-Stokes equations (1) were solved by means of a backward differentiation scheme with adaptive time stepping. The steady-state base flow configuration $\left\{\boldsymbol{u}_{0}, p_{0}\right\}(x, y, z)$ was solved through an iterative method, the Generalized Minimal RESiduals (GMRES), preconditioned using a standard multigrid algorithm. A convergence criterion of $10^{-3}$ is used for the relative error defined by a weighted euclidean norm for two successive iteration steps (see Correa et ll. $^{24}$ ). In the stability problem given in Eq. (2), the eigenvalues were computed employing a variant of the implicitly restarted Arnoldi method in the ARPACK routine. ${ }^{46}$ The junction domain was meshed with tetrahedral elements for the timedependent problem and hexahedral elements for the base flow and eigenvalue problems, with a higher concentration near the walls and in the crossing region of the $\mathrm{X}$-junction. We carried out convergence studies to estimate the minimal number of grid elements needed to obtain accurate results.

The accuracy of our numerical procedure is established by a grid convergence study over the leading eigenvalue (see Table I). Direct and adjoint eigenvalues, Eqs. (2) and (3), were obtained for four meshes with $A R=1$ and $L=7$. The difference among the eigenvalues is less than $1.3 \%$ for all the cases considered, so we used M3 in the rest of this study in order to achieve an accurate spatial description of the corresponding eigenmodes. The minimum size of the
TABLE I. Mesh convergence for the leading eigenvalues of the direct and adjoint problems with $A R=1, L=7$, and $R e=50$.

\begin{tabular}{lccc}
\hline \hline Mesh & Grid elements & $\lambda_{D}$ & $\lambda_{A}$ \\
\hline M1 & 10368 & 0.08021 & 0.07979 \\
M2 & 16072 & 0.07886 & 0.07988 \\
M3 & 22680 & 0.07828 & 0.07829 \\
M4 & 28800 & 0.07835 & 0.07802 \\
\hline \hline
\end{tabular}

TABLE II. Variation of the leading eigenvalue for different channel lengths, $L$, for $A R$ $=1$ and $R e=50$.

\begin{tabular}{cc}
\hline \hline$L$ & $\lambda_{D}$ \\
\hline 4 & 0.08102 \\
6 & 0.07887 \\
7 & 0.07889 \\
\hline \hline
\end{tabular}

domain was determined by analyzing the influence of the lengths of the channels on the value of $\lambda .{ }^{38}$ According to Table II, $L=6$ and $L=7$ result in accurate calculations of $\lambda$ and the corresponding eigenmode. Solving with $L=8$ shows negligible effects on the leading eigenvalue and the corresponding eigenmode. Choosing $L$ $=7$ is a good compromise between reliable results and computational cost to capture fully the dynamics of the flow and its instability.

We also validated the direct numerical simulation (DNS) codes and the stability analysis by considering the flow in a threedimensional T-shaped channel with two inlets and one outlet. This flow configuration was first studied experimentally and numerically by Engler et al. and Soleymani et al., respectively. ${ }^{47,48}$ Fani et al. studied the linear stability of the T-junction flow by means of a spectral element method. ${ }^{40}$ They reported a segregated flow regime for $R e$ $<175$ and a first flow bifurcation occurring at $R e=175$. For larger $R e$ 's, the symmetry of the flow is broken in an engulfment regime that mixes both incoming streams. We reproduced their results by means of the finite element method. The accuracy of the computation was controlled by a convergence study on the mesh parameters. A reliable result for the leading eigenvalues is obtained in a mesh domain of 23460 elements, finding $\sigma=-1.542 \times 10^{-2}$. This value differs by only $1.4 \%$ from the eigenvalue reported in Ref. 40 . Moreover, the shapes of the direct and adjoint modes associated with the leading eigenvalue match very well.

\section{RESULTS}

\section{A. DNS investigation of the flow structure}

We analyzed first the flow at low $R e$ values, for which the flow remains stable. For instance, for $R e=22$ [see Figs. 1 (b) and 1 (c)], the inlet flows are split into two equal parts after they meet in the crossing region and are in contact inside the outlet channels only in the plane $y=0$. This behavior is observed at small Reynolds numbers, independently of the value of the aspect ratio $A R>1$. Figure 1 (c) shows that this flow displays a noticeable extensional structure in the $z$ direction. 
For $R e>R e_{c}$, the segregated flow configuration becomes unstable and its symmetry is broken by the appearance of vortices with their axis parallel to the $x$ direction. Their formation is triggered by inertial effects, which let the incoming liquids cross the plane $y$ $=0$ (see below), resulting in a swirling motion toward the outlets. Figure 2 shows the streamlines in $\mathrm{X}$-junctions for $A R=1,6$, and 10 and $R e>R e_{c}$. A key result is that for $A R>3.8$ and $R e>R e_{c}$, more than one vortex appears in the flow. In this case, the incoming streams are sorted vertically in the outlets into alternating layers of the two pure fluids separated by zones where their streamlines are interlaced [these zones are located at $z$-coordinates similar to those of the vortex centers, as shown on the right-hand sides of Figs. 2(b) and 2(c)]. In the two latter cases, the total height of these zones of interlacement represents a smaller fraction of $H$ than for $A R=1$, as shown in Fig. 2(a) (right).

The number of layers is directly related to the number $n$ of vortices. As shown above, in the plane $y=0$, each vortex is located between stripes of two different fluids. Since there are fluid layers between both the upper and lower walls and the nearest vortices, the total number of layers is $n+1$. We note that the layered distribution of the two fluids is the same in both exits, with the stripes of a given fluid at the same location. After a transient flow settling phase, the flow field and the fluid distribution become timeindependent in the range of Reynolds numbers investigated. Quantitatively, we observed one single vortex for $A R \lesssim 3.8$, in agreement with Refs. 23 and 49, two vortices for $3.8 \lesssim A R \lesssim 8.5$, and three for $8.5 \lesssim A R<11$. For $A R=11$, which represents the highest demand for computational-power that we can deal with, four vortices appear.

Figures 3(a)-3(c) (Multimedia view) display the streamlines associated with the vortices in the middle plane $x=0$ of the junction, respectively, for one, two, and three vortices, and the multimedia view included in the figure displays their development with time. For $A R=1$, Fig. 3(a) the upward deflection of the blue fluid (in the figure) combined with the downward deflection of the orange one serves as a source for a counter-clockwise rotation, which propagates thereafter in the $z$ direction. For $A R=6$, Fig. 3(b) (Multimedia view), the orange flow is deflected toward the upper and lower walls of the channel, while the blue one is focused toward the middle. One has this time two sources of local rotation in opposite directions, which also propagate along $z$, creating two vortices of opposite circulations parallel to the direction of the outlet. The vortices result, therefore, from an inertia driven distortion of the initial separation plane $y=0$ of the two opposite flows encountered in the junction. If $A R$ increases further, more distortions appear, leading to more vortices, as shown in Fig. 3(c) (Multimedia view).

In Fig. 3(a) (Multimedia view), one notices that since the two fluids are identical, a configuration in which the orange fluid is deflected upward and the blue fluid downward is equally possible, leading to a clockwise rotating vortex. In the same way, in Fig. 3(b) (a)

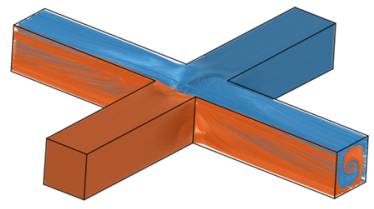

(b)

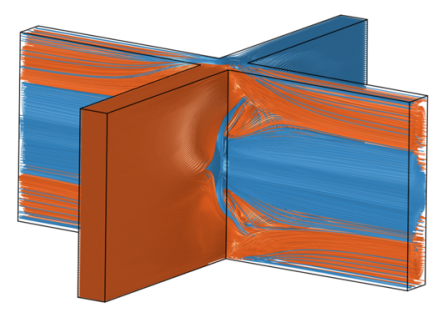

(c)

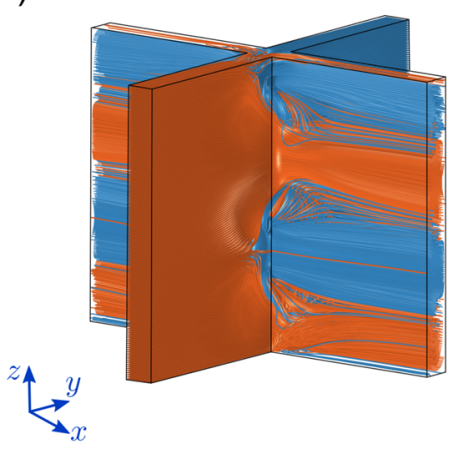

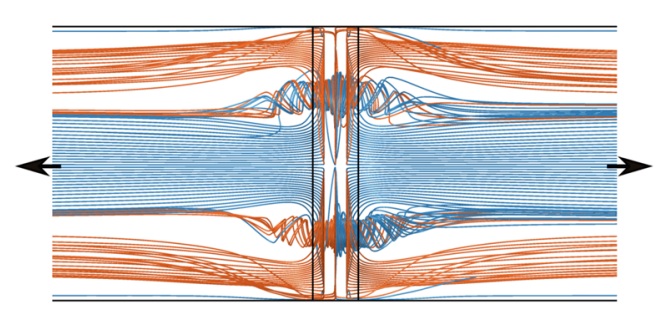
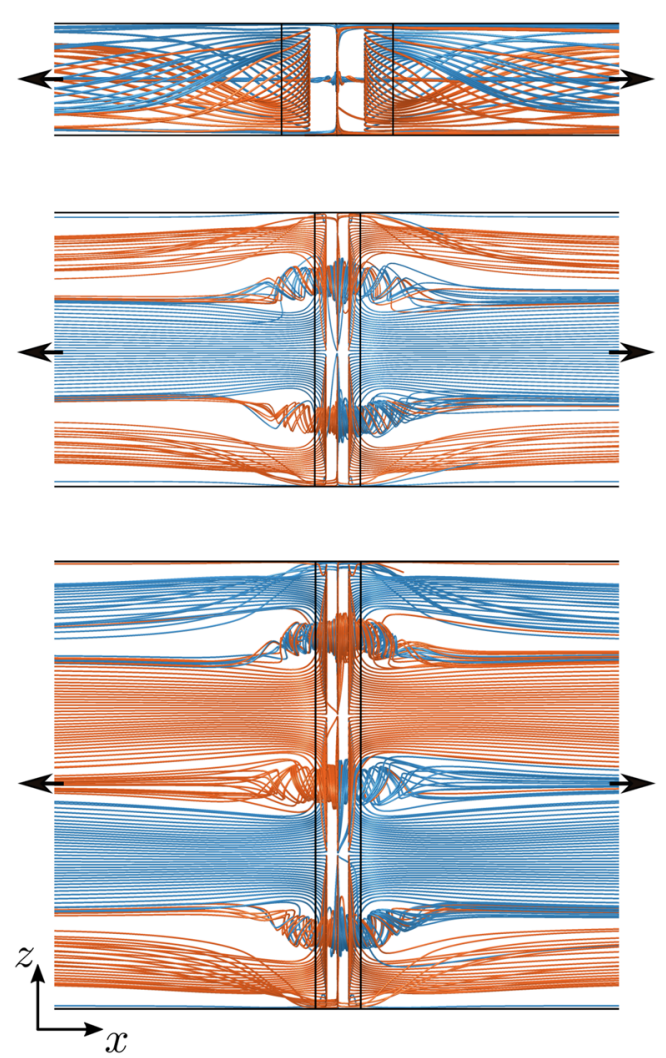

FIG. 2. Streamlines obtained by DNSs: (a) $A R=1$ and $R e=50$, (b) $A R=6$ and $R e=42$, and (c) $A R=10$ and $R e=44$. The blue and orange colors indicate liquids coming from each inlet. Left: 3D perspective views; right: plane cut showing representative streamlines. 
(a)

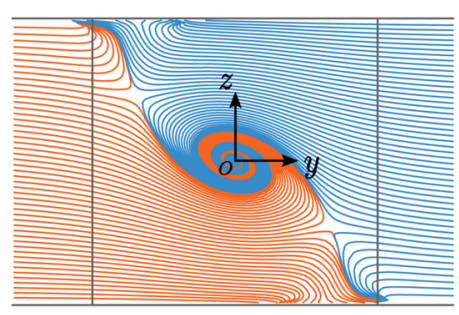

(d)

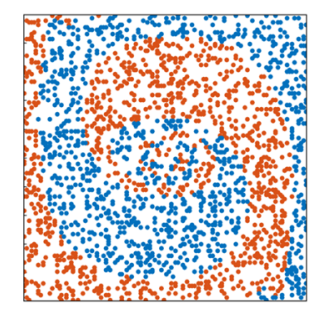

(g)

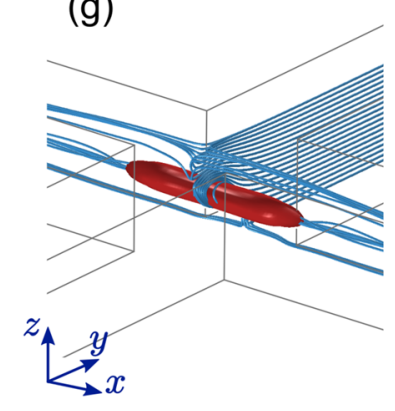

(b)

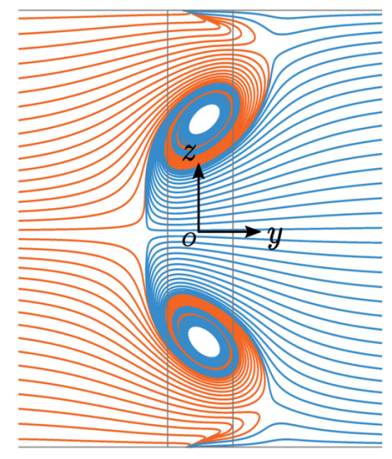

(e)

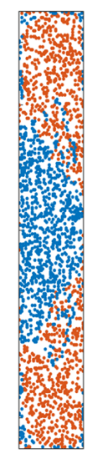

(h)

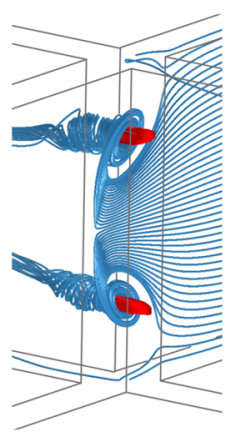

(c)

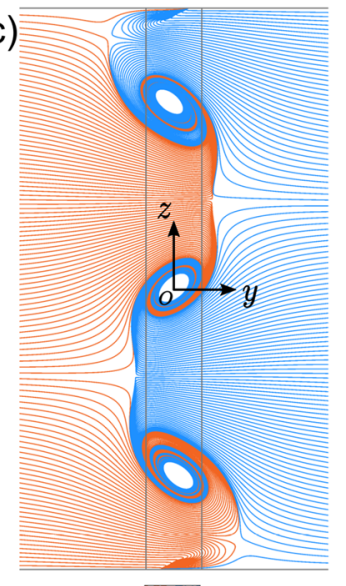

(f)

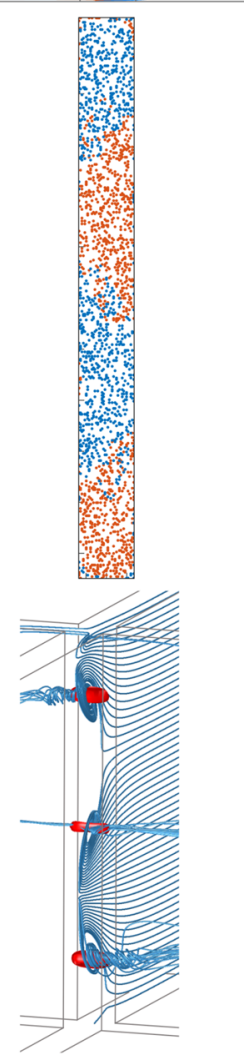

FIG. 3. Streamlines in the plane $x=0$ for the same DNS as in Fig. 2 with (a) $A R$ $=1, R e=50$, (b) $A R=6, R e=42$, and (c) $A R=10, R e=44$. Vertical lines: location of outlets walls. (d)-(f) Flow line distribution for the same experiments in the section $x=7$ of the outlet channel. Streamlines intersect the section at a right angle and appear as dots. (g)-(i) $3 D$ views of $Q$ isosurfaces (in red) for the same three experiments. In blue: streamlines from one entrance. Multimedia view: development of streamlines with time in the plane $x=0$ for these experiments. $R e$ increases at first linearly with time from $1(t=0)$ to the $R e$ values corresponding to cases (a)-(c) reached, respectively, for $t=100,50$, and 50, and remaining constant afterward. Multimedia view: https://doi.org/10.1063/5.0026829.1

(Multimedia view), the orange fluid might as well advance further at the center and the blue fluid near the walls. This, too, would result in a reversal of the two vortices with an orange fluid layer in the center of the section and blue layers near the ends. Similarly, for other values of $A R$, one may swap the locations of the two fluids in the outlet channels provided the rotation of the vortices is also reversed. Experimentally, both configurations should be observed with equal probabilities.

The effect of the transit of the liquid through the output channels is demonstrated by comparing Figs. 3(a)-3(c) $(x=0)$ and

Figs. 3(d)-3(f) $(x=7)$. For all $A R$ 's, the vortical flow is limited to the region close to the center of the section at $x=0$. Nevertheless, the aspect ratio affects the distribution of the two fluids at the outlets. For $A R=1$, Fig. 3(d), the blue-orange streamlines define a helical-shaped interface that is longer than that observed in the layered fluid distribution for $A R=6$ and $A R=10$ shown in Figs. 3(e) and $3(\mathrm{f})$, respectively. The vorticity distribution at the center differs, therefore, strongly from that at the outlet. We identify the $3 D$ structure of the vortices by $Q$-contours. ${ }^{26,36,50,51}$ The criterion $Q$ is defined as $Q=\frac{1}{2}\left(\|\boldsymbol{\Omega}\|_{F}^{2}-\|\boldsymbol{S}\|_{F}^{2}\right)$, where $\boldsymbol{\Omega}=\frac{1}{2}\left(\nabla \boldsymbol{u}-(\nabla \boldsymbol{u})^{\top}\right)$ and 
$\mathbf{S}=\frac{1}{2}\left(\nabla \boldsymbol{u}+(\nabla \boldsymbol{u})^{\top}\right)$ are the rotation and the strain-rate tensors, respectively, and $\left(\|\cdot\|_{F}\right)$ stands for the Frobenius norm. In Figs. 3(g)3(i), $Q$ isosurfaces are represented in red for $A R=1,6$, and 10; in these three cases, the vortices develop only in the region where the channels meet and hardly penetrate into the outlet channels. As a result, close to the exits, the $x$-component of the vorticity disappears and the parabolic profile and its vorticity distribution are recovered.

The physical mechanism of the development of the vortices is the same for all $A R$ 's analyzed; it is illustrated for $A R=6$ in the time sequence of Figs. 4(a)-4(f) and for $A R=1,6$, and 10 in the multimedia views of Figs. 3 and 4 by maps of the $x$-component of the vorticity and streamlines. Initially, in Fig. 4(a), the vorticity is non-zero only close to the upper and lower walls and the flow lines are straight and horizontal. In Fig. 4(b) after $R e$ has increased with time, the vorticity has diffused from the top and bottom walls toward $z=0$, especially in the region close to the interface. In Fig. 4(c), four Dean cells start to develop on both walls of the intersection with an inversion of the sign of the vorticity close to these walls. In Figs. 4(d) and $4(\mathrm{e})$, the largest vorticity keeps developing close to these walls and the flow symmetry is lost. Finally, the steady state corresponding to Fig. 3(b) is reached in Fig. 4(f). The key influence on the (a)

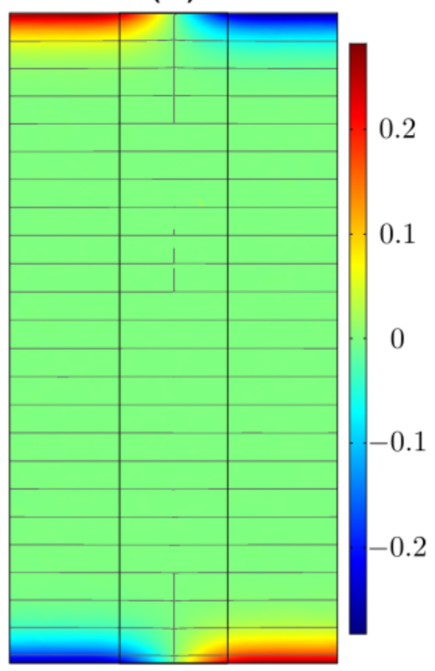

(d)

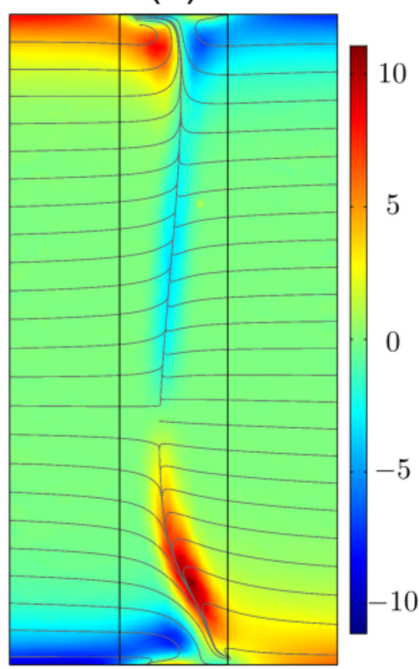

(b)

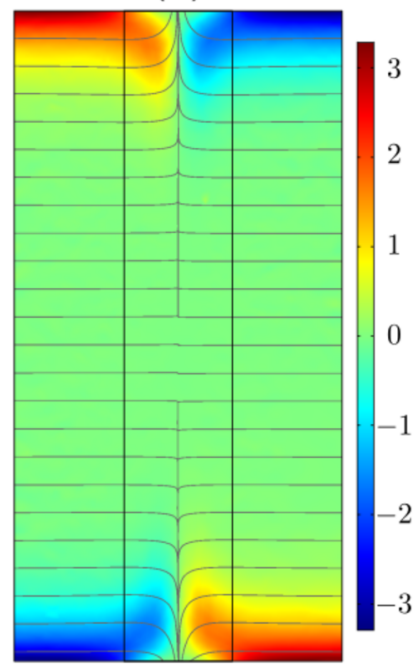

(e)

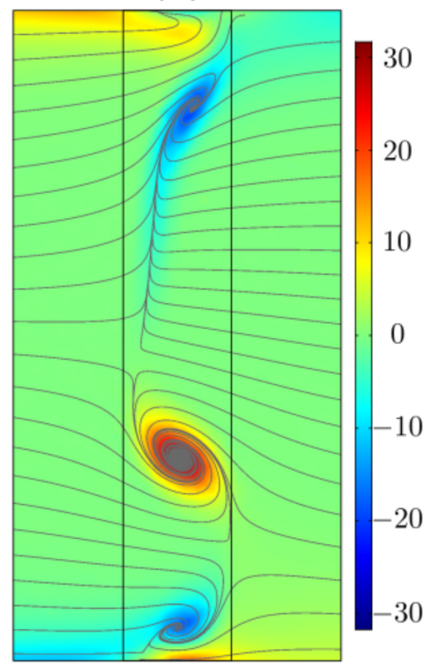

(c)

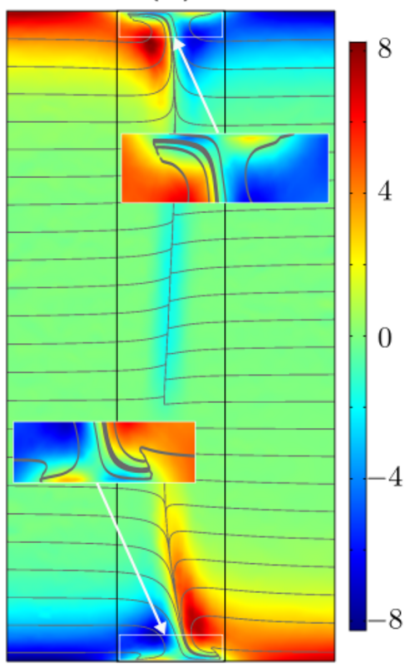

(f)

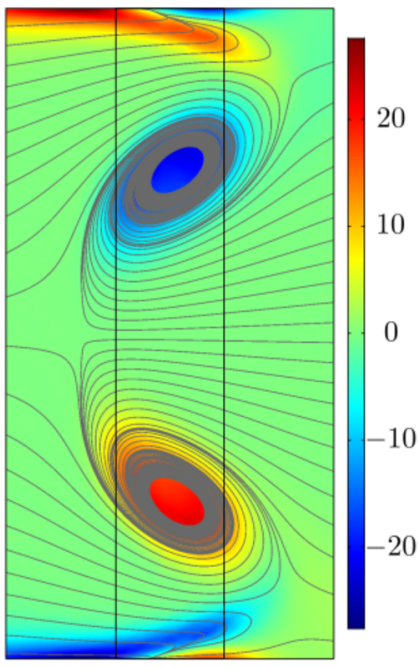

FIG. 4. Time sequence of maps of the $x$-vorticity component (color scale) in the plane $x=0$ for the same experiment as in Fig. $3(b)$ (Multimedia view) with $A R=6$ and $R e$ increasing linearly with time from $R e=1(t=0)$ to $R e=42(t=50)$ and remaining constant thereafter. Black lines: streamlines. (a) $t=0$, (b) $t=20$, (c) $t=60$ (insets: local inversion of the sign of the vorticity), (d) $t=80$, (e) $t=94$, and (f) $t=130$. Multimedia view: development of the $x$-vorticity with time in the plane $x=0$ for the same three experiments as in Fig. 3 (Multimedia view). Multimedia view: https://doi.org/10.1063/5.0026829.2 


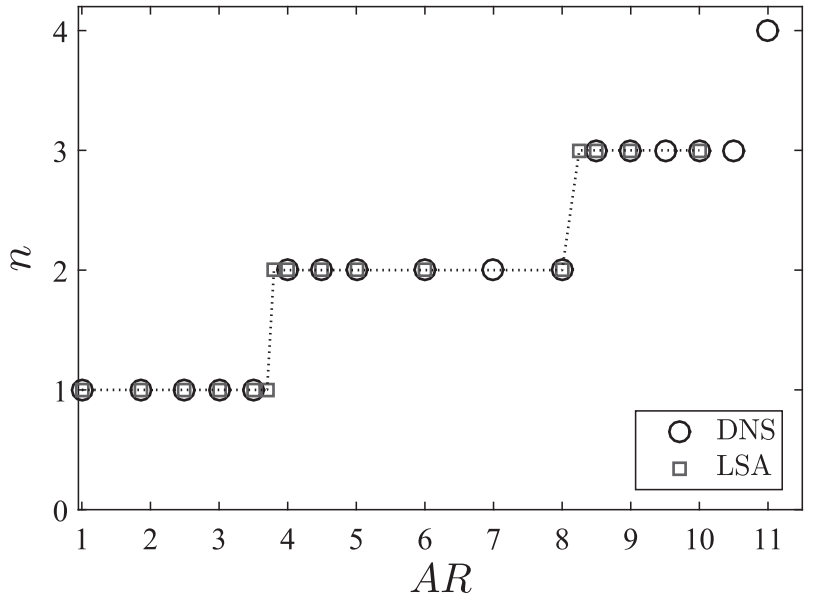

FIG. 5. Number $n$ of vortices as a function of the aspect ratio $A R$. Results from DNSs: $\bigcirc$; results from linear stability analysis (LSA): $\square$ (see Sec. III B for explanations).

triggering of the instability of the formation of the Dean vortices and the diffusion of the $x$-component of the vorticity was first explained for $A R=1$ by Haward et al. ${ }^{23}$ and Burshtein et al. ${ }^{21}$ Figures 3 and 4 (Multimedia views) display this same transition from zero to $n$ vortices, respectively, for the streamlines and the vorticity for $A R=1$, 6 , and 10. We will show in Sec. III B that the regions with the maximum value for the sensitivity $S$ develop four local $x$-vorticity maxima (close to the upper and lower walls) for $z \pm 1.6$ (in the present case). Then, the actuation on these regions may hamper or boost vorticity generation.

The first important result of the simulations is that the number $n$ of vortices observed depends only on the aspect ratio $A R$ and not on $R e$ provided $R e>R e_{c}\left(R e_{c}\right.$ depends on $\left.A R\right)$. The variation of $n$ with $A R$ is shown in Fig. 5; one observes a stepwise increase with no overlap between the different steps.
Figure 6 displays the variations of the $y$-component of the velocity along the $z$-axis $(x=0, y=0)$ for different $A R$ values, for which $n=2$ [Fig. 6(a)] and $n=3$ [Fig. 6(b)] vortices are observed. The very good collapse of the normalized velocity profiles $\left(v_{\max , z}\right.$ is the maximum absolute value of $v$ along the $z$-axis) in each graph implies that the normalized distance $d_{v} / H$ between the centers of two adjacent vortices is nearly constant with $A R$ : one has $d_{v} / H \simeq$ 0.5 for $n=2$ and $d_{v} / H \simeq 0.33$ for $n=3$. Therefore, the simulations suggest that the vortices are equidistant along the $z$-axis; the distance $d_{v} / H$ between two adjacent vortex centers is $\simeq 1 / n$, and the distance of the top and bottom vortices to the adjacent walls is $\simeq 1 / 2 n$.

In the same way, we performed these simulations at different $R e$ 's $\left(\geq R e_{c}\right)$ for $A R=6$ (respectively, 10) corresponding to $n=2$ (respectively, 3) vortices. We also included one case with $A R=11$, for which one observes four vortices; further increments of $A R$ are beyond our current computing capabilities. Figure 7 (a) displays, in the two cases, the distances between the different vortices as a function of Re: their relative variation is less than $5 \%$. Figure 7 (b) shows the variation of the spacing $d_{v}$ of adjacent vortices (averaged over several pairs of vortices when $n \geq 2$ ) with $A R$. Note that the variations of $d_{v}$ with $A R$ are consistent with the estimation $d_{v} \simeq A R / n$ [dashed lines in Fig. 7(b)] based on the order of magnitude: $d_{v} / H$ $\simeq 1 / n$, mentioned above.

The onset of the instability is analyzed in Fig. 8. To characterize it, we selected $v_{\max , z}$ as the order parameter and explored its variation with $R e$ for three different $A R$ 's. ${ }^{23}$ These curves were obtained by means of numerical simulations using increasing and decreasing ramps of $R e$ with steps $\delta R e=1$ in the vicinity of the critical value $R e_{c}$. The segregated stable flow is characterized by $v_{\max , z}=0$, which means that the interface between the two fluids remains in the plane $y=0$. The sudden increase in $v_{\max , z}$ indicates the onset of the instability. As can be seen from the three examples in Fig. 8, there is an hysteresis for all values of the number $n$ of vortices. The critical Re's for the increasing ramps are $R e_{c}=44,37$, and 41, and for the decreasing ramps, $R e_{c}^{*}=41,30$, and 36 , for $A R=1,6$, and 10 , respectively. The fact that $R e_{c}^{*}<R e_{c}$ for all the explored values of $A R$ implies, therefore, that the instability leading to the appearance of vortices is subcritical in all these cases.
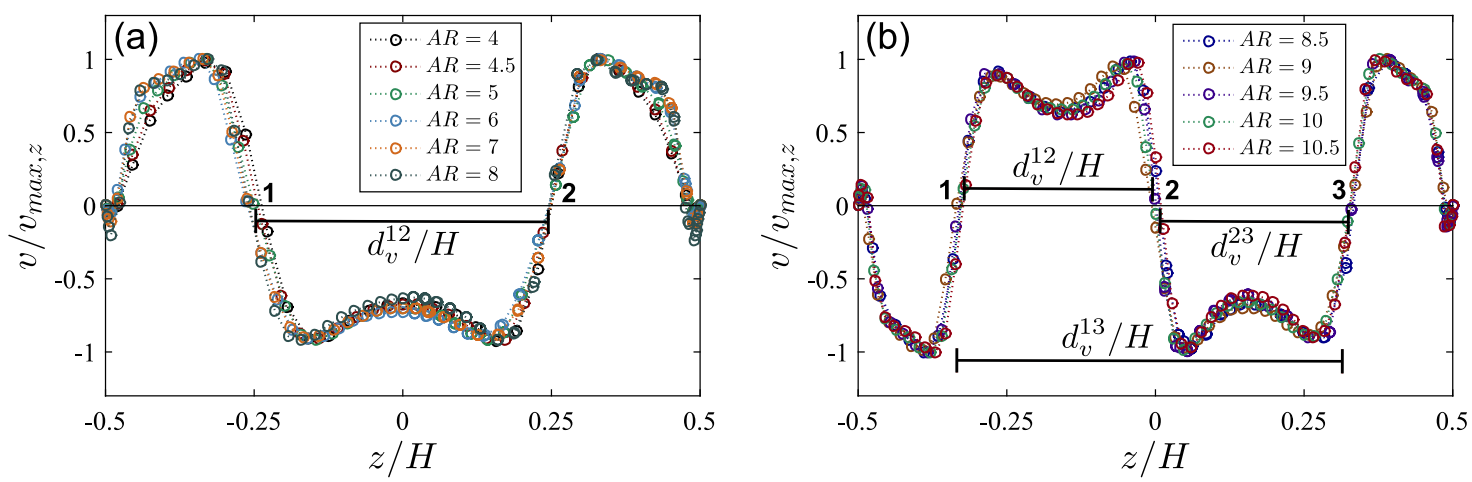

FIG. 6. Variation of the normalized $y$-velocity component $v / v_{\max , z}$ with $z / H$ along the $z$-axis $(x=0, y=0)$ for several aspect ratios $A R$ and $R e=44$. Profiles correspond to (a) $n=2$ and (b) $n=3$. Boldface numbers: labels for vortex centers; $d_{v}^{i j} / H=$ normalized distance between the centers of vortices $i$ and $j$. 

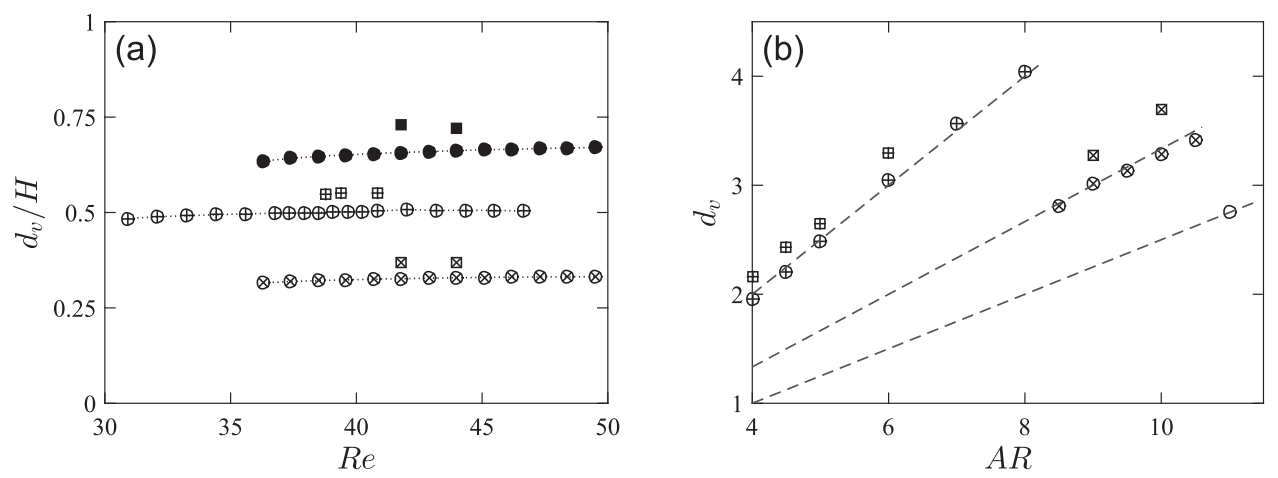

FIG. 7. (a) Normalized distances $d_{v} / H$ between vortices as a function of $R e$ $>R e_{c}$. Circles correspond to DNSs (squares for linear stability analysis, Sec. III B). For $A R=6, d_{v}=d_{v}^{12}: \oplus(\boxplus)$, and for $A R=10, d_{v}=\left(d_{v}^{12}+d_{v}^{23}\right) / 2: \otimes$

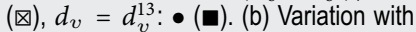
$A R$ of the mean of the dimensionless distances $d_{v}^{i, i+1}$ between adjacent vortices for different numbers $n$ of vortices from DNSs (linear stability analysis). $n=2: \oplus$ $(\boxplus) ; n=3: \otimes(\otimes) ; n=4: \bigcirc$. Dashed lines: variations as $A R / n$.
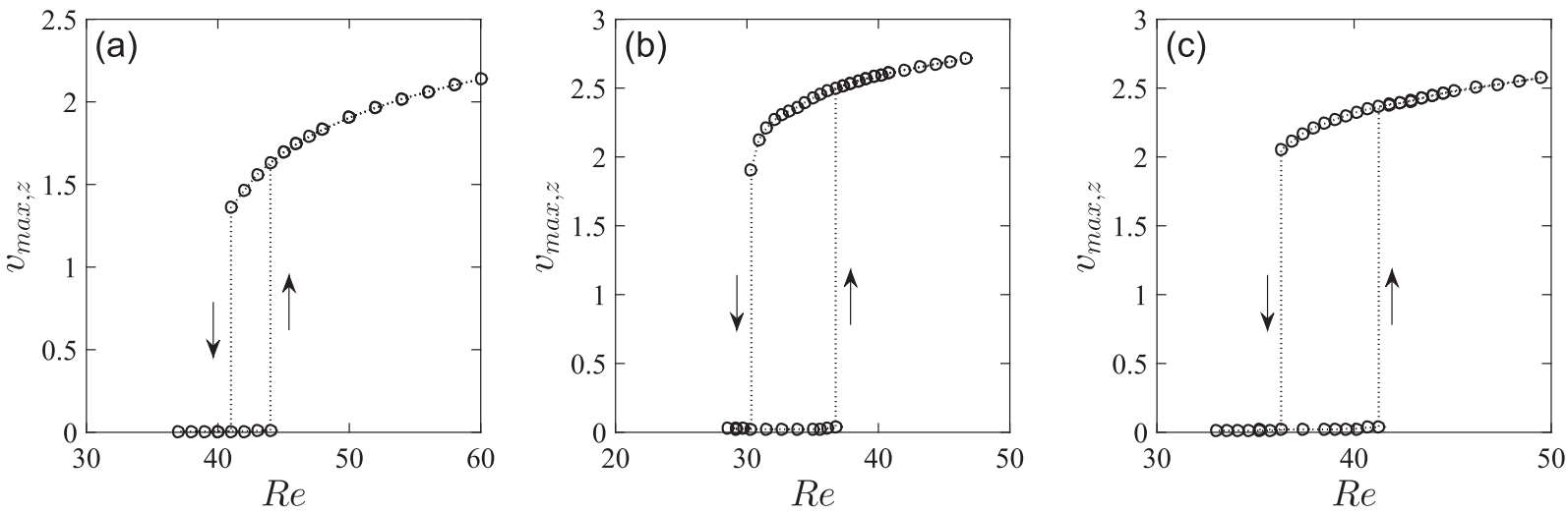

FIG. 8. Maximum $v_{\max , z}$, along the $z$-axis, of the velocity component $v$ as a function of $R e$. In (a)-(c), the instability corresponds to the appearance of one $(A R=1)$, two (AR $=6)$, and three $(A R=10)$ vortices, respectively.

\section{B. Linear stability analysis}

In this section, we investigate the global stability of the flow and compare the results, such as the values of $R e_{c}$ and the number of vortices and their spacing, to those reported in Sec. III A for the same aspect ratios $A R$. The global stability of the flow is analyzed by the computation of the eigenvalues and eigenmodes of the direct problem [Eq. (2)]. The natural choice of the base flow required in the calculation is the steady-state symmetric segregated flow field. In order to obtain the latter for $R e$ higher than $R e_{c}$, we compute the solution in half of the domain and impose a symmetry boundary condition in the plane $y=0$. The solution is then mirrored to the other half of the domain.

A discrete set of eigenvalues is shown in Fig. 9 for stable (a) and unstable (b) flows with $A R=1$. The excellent agreement between the direct and adjoint spectra demonstrates the reliability of the (a)

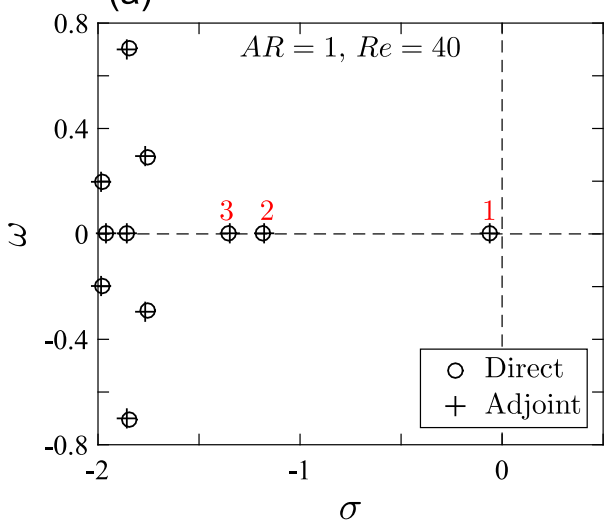

(b)

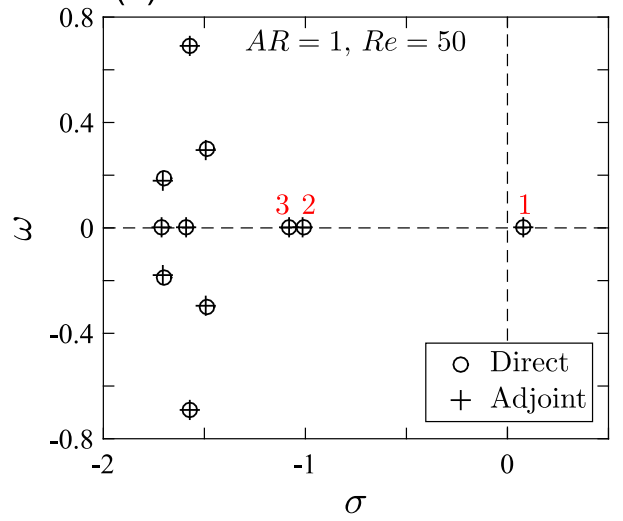

FIG. 9. Eigenvalue spectrum for the direct $(\bigcirc)$ and adjoint (+) problems for $A R=1$ and (a) $R e=40<R e_{c}$ and (b) $R e$ $=50>R e_{c}$. The horizontal and vertical axes correspond, respectively, to the real $(\sigma)$ and imaginary $(\omega)$ parts of the eigenvalue. Red numbers above the leading eigenvalues are the number of vortices in the corresponding eigenmodes. 


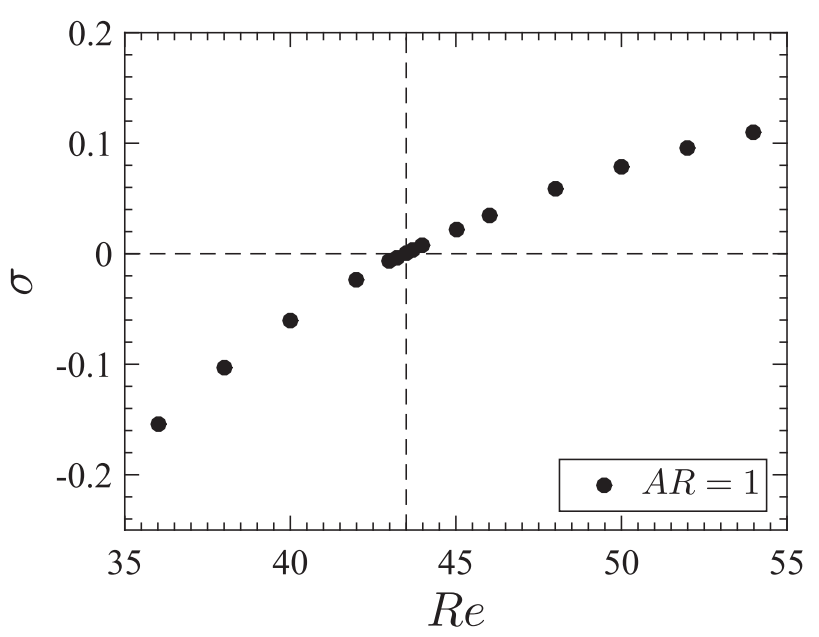

FIG. 10. Real part of the leading eigenvalue as a function of $R e$ for $A R=1$. The vertical dashed line corresponds to the critical Reynolds number $R_{c}=43.5$.

numerical procedure. The eigenvalues $\lambda=\sigma+i \omega$ are distributed symmetrically with respect to the real axis. In all the simulations, the eigenvalue with the largest growth rate $\sigma$ corresponds to a steadystate perturbation $(\omega=0)$. This is also the case for the two other eigenvalues with $\sigma$ closest to 0 , labeled 2 and 3 in Fig. 9.

The change in the growth rate $\sigma$ of the leading eigenvalue from negative to positive shown in Fig. 10 allows us to determine precisely the critical Reynolds number, which is found to be $R e_{c}=43.5$ \pm 0.15 . The three-dimensional global mode associated with the leading eigenvalue (labeled 1 in Fig. 9) is shown in Fig. 11 for $R e=50$. The eigenmodes are spatially located mainly in the outlet pipes. The $\hat{v}$ and $\hat{w}$ components are composed of two main lobes, both symmetrical with respect to the plane $x=0$ and with a maximum of intensity in the intersection (see arrows in Fig. 12). On the other hand, $\hat{u}$ consists of four lobes, anti-symmetrical with respect to the plane $x=0$, with their highest and lowest intensities outside the junction, at a distance $x= \pm 1.7$ (see Fig. 12).

The velocity field of the leading eigenmode is displayed in Fig. 12 in four sections of an outlet branch. Similar to the corresponding DNS, one observes in these $(y, z)$ cut planes a single vortical motion around the junction center. Although all perturbation components are of the same order of magnitude, $\hat{v}$ is $\sim 2.5$ times higher than $\hat{u}$ and $\hat{w}$. The maximum of $\hat{v}$ occurs at $(x, y, z)=(0$, $0, \pm 0.25)$ where $(\hat{u}, \hat{w})=(0,0)$. The component $\hat{w}$ reaches its maximum value in the plane $z=0$ near the lateral walls, at the beginning of the outlet branches $(x= \pm 0.5)$.

The stability has also been studied for the junctions with $A R$ $=6$ and 10, and the results are compared to those of the DNSs. Like for $A R=1$, the leading eigenvalues are real and their variation with $R e$ is shown in Fig. 13. The critical Reynolds numbers are, respectively, $R e_{c}=39 \pm 0.15$ and $42.4 \pm 0.1$ for $A R=6$ and 10 .

Figure 14 displays isosurfaces of the velocity perturbation components of the mode associated with the leading eigenvalue for $A R$ and $R e$ values corresponding to two, Figs. 14(a)-14(c), and three, Figs. 14(d)-14(f), vortices. In these cases, like for $A R=1$, the values of $\hat{v}$ and $\hat{w}$ are largest inside the intersection and the maximum for $\hat{u}$ lies in the outlet branches for $\hat{u}$. In addition, $\hat{u}$ remains antisymmetric with respect to the plane $x=0$, while $\hat{v}$ and $\hat{w}$ are still symmetric. There are, however, significant changes compared to the case $A R$ $=1$. For $\hat{u}$, the group of four lobes in each outlet channel is split, for $A R=6$, into two distant groups with a new pair of lobes at half height, while for $A R=10$, an additional pair appears. For $\hat{v}$, there are three lobes for $A R=6$, instead of two for $A R=1$, and they are less elongated along $x$; for $A R=10$, four elongated lobes are obtained. (a)

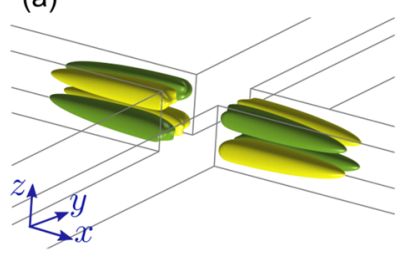

(b)

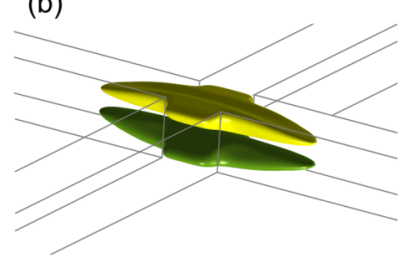

(c)

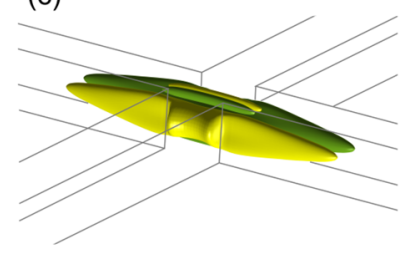

FIG. 11. Isosurfaces for $A R=1$ and $R e$ $=50$ of the $\hat{\boldsymbol{u}}$ components: (a) $\hat{u}$, (b) $\hat{v}$, and (c) $\hat{w}$. Yellow corresponds to the value $-10^{-6}$ and green to $10^{-6}$.
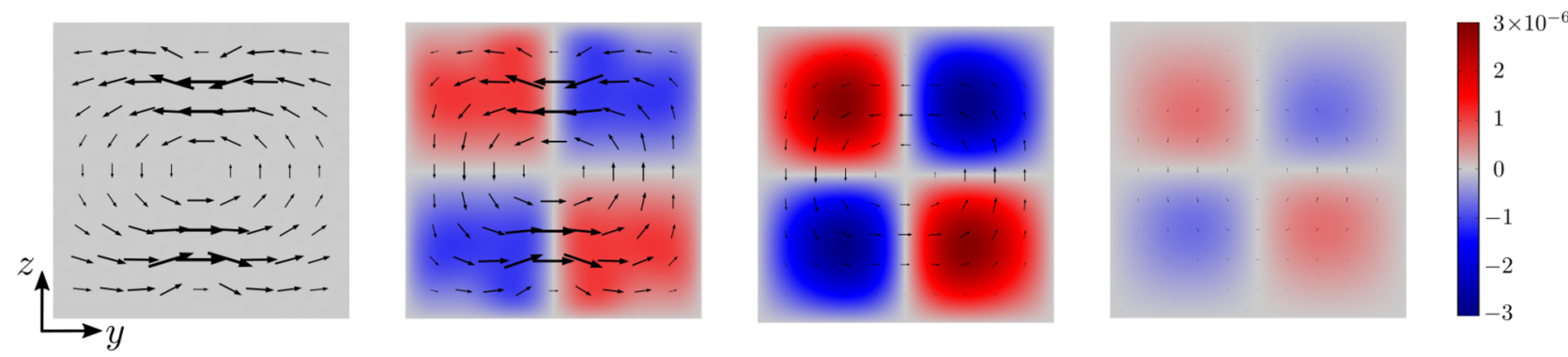

FIG. 12. Perturbation solution in $y z$-planes for $A R=1$ and $R e=50$ at several distances $x$ along an outlet branch (from left to right: $x=0,0.5,1.5$, and 3 ). The length of the vectors is given by the values of the components $\hat{v}$ and $\hat{w}$, and colors code the component $\hat{u}$. 

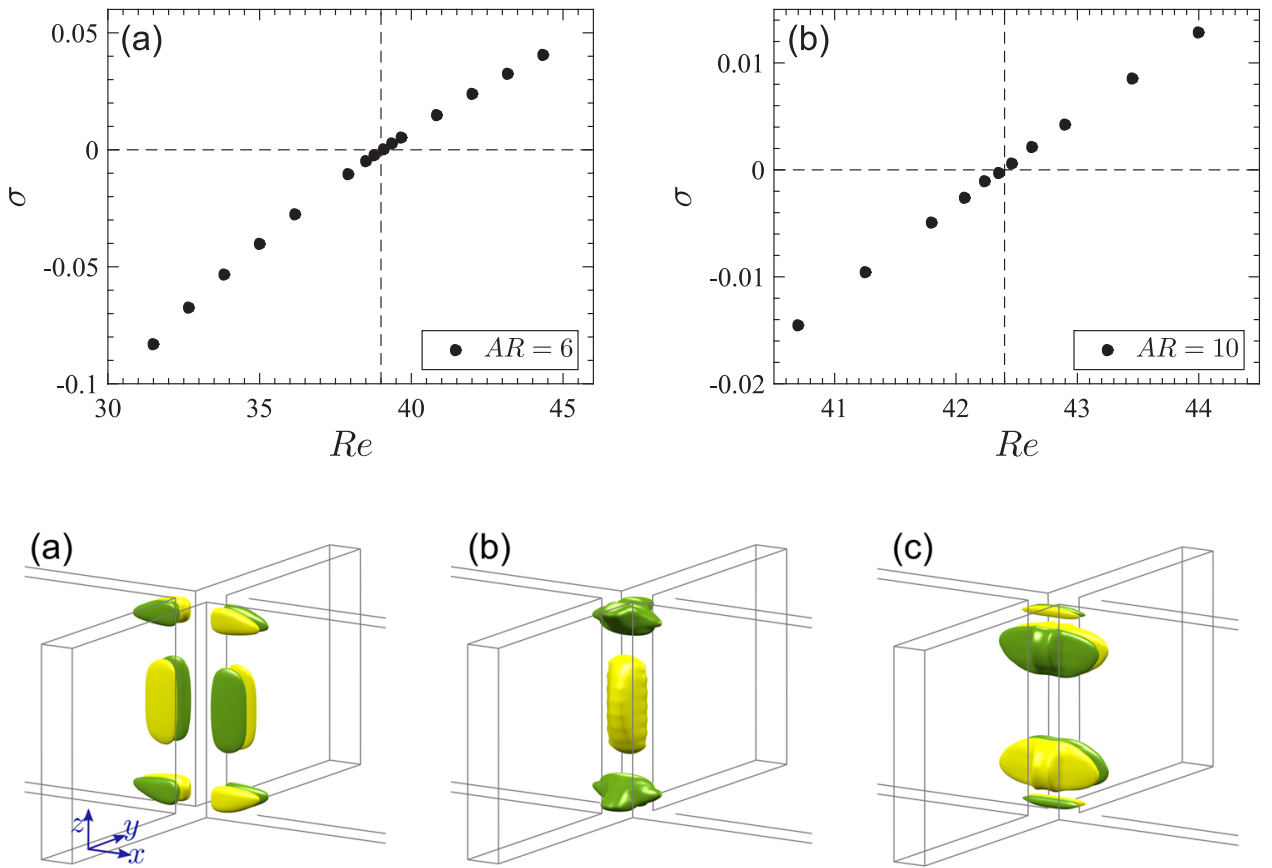

(d)

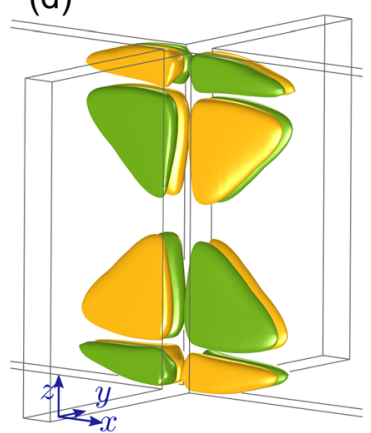

(e)

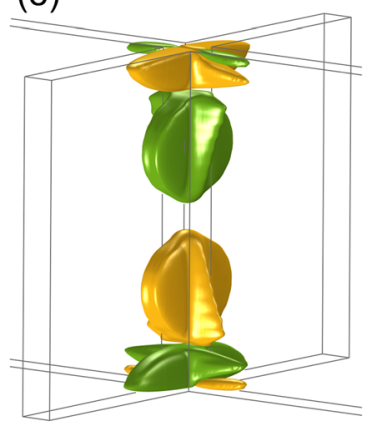

(c)

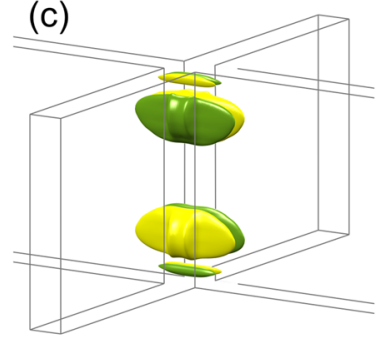

(f)

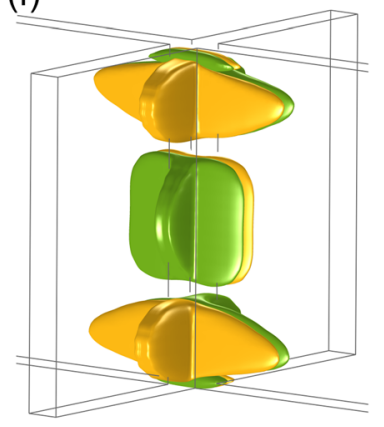

FIG. 13. Real part of leading eigenvalues vs $R$ e for (a) $A R=6$ and (b) $A R=10$. Critical Reynolds numbers are, respectively, $R e_{c}=39$ and $R e_{c}=42.4$
FIG. 14. Isosurfaces of the velocity perturbation components. Top: $A R=6, R e$ $=42$. (a) $\hat{u}$, (b) $\hat{v}$, and (c) $\hat{w}$. Yellow (green) corresponds to the value $-3 \times$ $10^{-6}\left(3 \times 10^{-6}\right)$. Bottom: $A R=10, R e=$ 44. (d) $\hat{u}$, (e) $\hat{v}$, and (f) $\hat{w}$. Yellow (green) corresponds to the value $-1.5 \times 10^{-6}$ $\left(1.5 \times 10^{-6}\right)$.
For $\hat{w}$, there are, for $A R=6$, four pairs of medium size, mildly elongated lobes and two small, instead of two large, very elongated ones; for $A R=10$, there are two more lobes and they are more elongated. Regarding the symmetry of the lobes with respect to the plane $z=$ 0 , it is determined by the even $(n=2$ for $A R=6)$ or odd $[n=1$ (respectively, 3) for $A R=1$ (respectively, 10)] values of the number of vortices.

In Fig. 15, the three velocity perturbation components of the corresponding leading eigenmode for each $A R$ are presented at different distances $x$ along the outlet. For $A R=6$, Fig. 15(a), the field $(\hat{v}, \hat{w})$ displays a double vortex structure in the $x=0$ plane as in the DNS velocity field of Fig. 3(b) (Multimedia view). In the central region, the perturbation points directly toward the left inlet, extending slightly beyond the section shown in Fig. 15, while the vortices are distributed in the upper and lower portions of the plane. For $A R=10$, Fig. 15(b), one observes, as expected, three vortices like in Fig. 3(c) (Multimedia view). For both $A R$ 's, the $\hat{v}$ and $\hat{w}$ modes decay slowly along the outlet branch of the junction and $\hat{u}$ changes sign at the plane $y=0$ and at the height $z$ at which a vortex center is located. For $A R=6$, the absolute value of $\hat{u}$ increases from the center and is maximal at approximately $x=1$, while for $A R=10$, it is highest at approximately $x=1.5$. For $A R=6$ and 10 , one finds, like for $A R=1$, that the three eigenvalues closer to $\sigma=0$ correspond to eigenmodes with one, two, or three vortices (see Fig. 9); however, only one of these three eigenvalues may become positive when $R e$ increases and corresponds to the number $n$ of vortices depending only on $A R$.

In Fig. 16, we compare the variations of $\operatorname{Re}_{c}$ with $A R(1<A R$ $<10$ ) obtained from the linear stability analysis (LSA) using Eq. (2) to those determined from DNSs. The values of $R e_{c}$ found by the stability analysis are in good agreement with those of the DNS obtained by using an increasing ramp for $R e$ and display, like them, an initial linear increase in $R e_{c}$ for $A R \gtrsim 2$, leveling off for $A R \geq 5$. This suggests that at large values of $A R, \operatorname{Re}_{c}$ increases only slowly with $H$; Ref. 34 reports indeed a critical value $R e_{c}=55$ at $A R=262$ for increasing flow rates and $R e_{c}=43$ for decreasing ones. The minimum of $R e_{c}$ is found at approximately $A R=2$, so that, for $R e<$ 
(a)

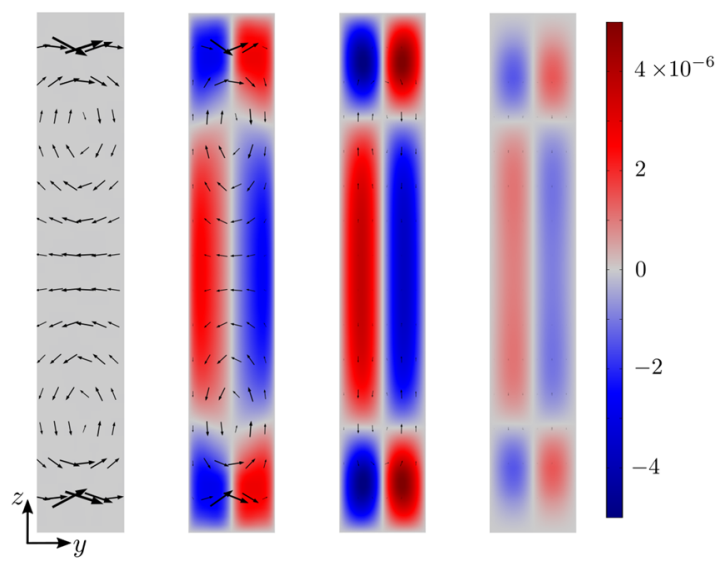

(b)

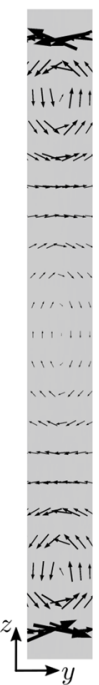

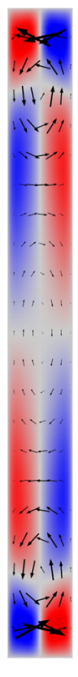
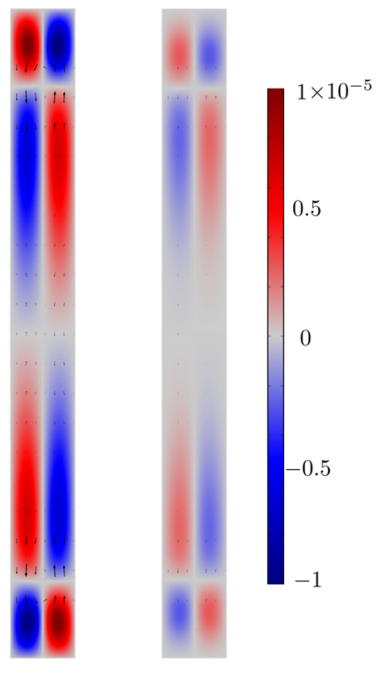

FIG. 15. Map of the velocity perturbation components $(\hat{u}, \hat{v}, \hat{w})$ of the leading eigenmode in different sections $x=c s t$ for (a) $A R=6, R e=42$ and $(\mathrm{b}) A R=10, R e=44$. Black arrows correspond to $(\hat{v}, \hat{w})$, and the color map codes the values of $\hat{u}$.

23, the flow is completely segregated within the range of $A R$ values analyzed. The results for both increasing and decreasing $R e$ ramps compare well with those of Haward et al., ${ }^{23}$ who reported an increase in $R e_{c}$ for decreasing $A R$ when $A R \lesssim 2$. The numbers of vortices as a function of $A R$ from the linear analysis have been superimposed in Fig. 5 onto the equivalent data points from the DNS. The results are perfectly compatible, and the transition from $n$ to $n+1$ vortices can be determined more precisely by means of the linear analysis due to the reduced computation time.

Let us compare now the spatial structure of these modes to the single and multiple vortex instabilities of the nonlinear DNS results. As shown above, the leading mode that dictates the shape of the base flow instability clearly captures the number of vortices predicted by

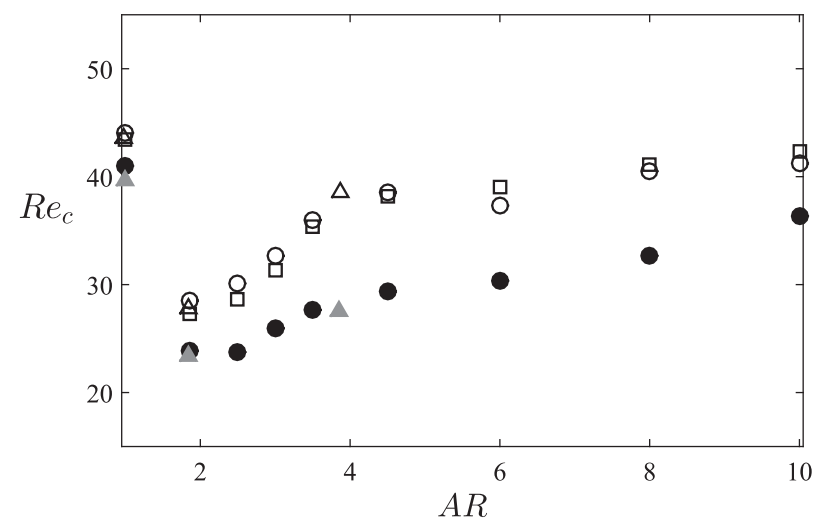

FIG. 16. Critical Reynolds numbers $R e_{c}$ as a function of $A R$ : open (respectively, filled) symbols for increasing (respectively, decreasing) Re's. DNS: $\bigcirc(\bullet)$; linear stability analysis (LSA): $\square$; Haward et al..$^{23}$ [inset of Fig. 2(i)]: $\Delta(\Delta)$. the DNS. The distances between adjacent vortices arising from the two methods have been superimposed in Fig. 7: the values obtained from the linear analysis are slightly higher $(5 \%)$ that those from the DNS. One notes, however, that while accurate $R e_{c}$ values may be expected from the linear analysis, the spacing between vortices is approximated since, in the DNS, the instability is already developed and the flow structure may be influenced by nonlinear terms.

Moreover, the null frequency component $\omega$ of the leading eigenvalues is compatible with the steady state of the flow obtained for $R e>R e_{c}$ by means of the direct numerical simulations. The linear stability analysis indicates, indeed, that the instability of the flow occurs through a pitchfork bifurcation. Additionally, the global mode structures suggest in all cases that the effect of inertia is higher in the intersection of the channels, as shown by the large values of the component $\hat{v}$. The accommodation of the vortex flow in the downstream direction leads to a strong perturbation of the velocity component $u_{0}$ along the outlet branches. Finally, we observe that when $R e$ increases, there is a spatial elongation of all eigenmodes toward the outlets.

\section{Sensitivity function}

We investigate now the spatial variations of the sensitivity function $S$ defined by Eq. (4) for flows in which axial vortices are present. The occurrence and location of regions of large sensitivity to local feedback forces is indeed closely related to the global mode dynamics. ${ }^{42}$ For instance, if variations of the flow are induced in regions of low $S$ values, this influences very little the leading eigenvalue. Any strategy aimed at controlling the instability must, therefore, be applied to a region of the flow where $S$ is large.

Figure 17 displays isosurfaces corresponding to several normalized values of the sensitivity $S$ for the cases $A R=1$ (a) and $A R=6$ (b) studied above; the results obtained for $A R=10$ are not shown for 
(a)
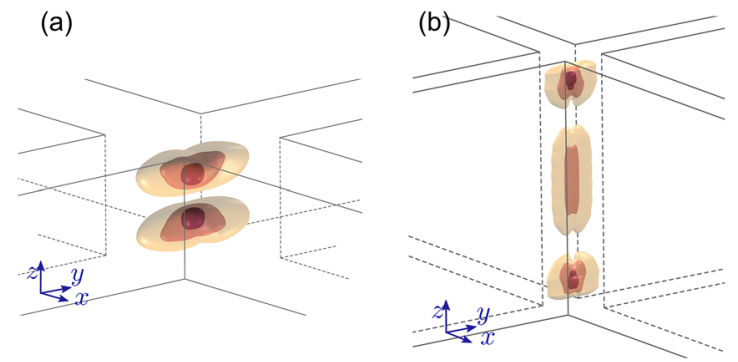

FIG. 17. Maps of isosurfaces of the sensitivity function $S$ for (a) $A R=1, R e=50$ and (b) $A R=6, R e=42$. Isosurfaces shown correspond to $S=0.25$ (yellow), $S$ $=0.5$ (orange), and $S=0.75$ (red). concision but will be briefly mentioned below. The values of $S$ for the three isosurfaces shown are $S=0.25,0.5$, and 0.75 , and the outer (inner) shells correspond to the smaller (higher) sensitivities.

For $A R=1$, Fig. 17(a), $S$ is largest inside two symmetrical regions of the crossing zone of the junction elongated toward the inlets. More precisely, the maximum of $S$ is located on the $z$-axis at $z / H \approx \pm 0.15$. The inspection of the data shows that these two points are closer to the center of the junction than the locations of the maxima of $w_{0}$ and $\hat{v}$. We also observe that the region where the sensitivity is highest differs from the location of the vortex $(z=0)$. The latter feature resembles the result of Chen $e t a l .{ }^{36}$ for T-junctions, where the sensitivity is highest in lobes located in the exterior region of the vortices. However, in the geometry of the latter work, the $S$ lobes are elongated in the direction of the outlets instead of the inlets, (a)

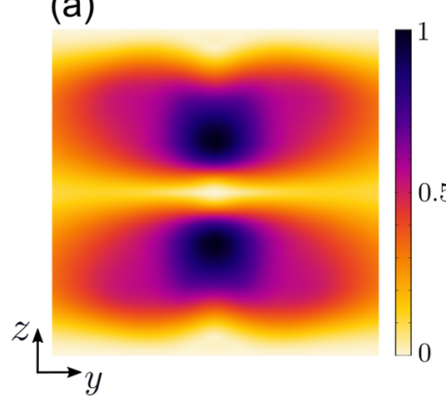

(d)

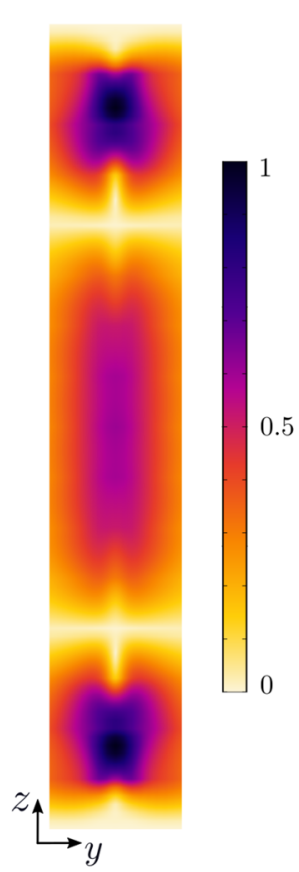

(b)

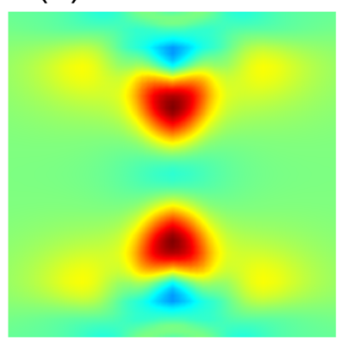

(e)

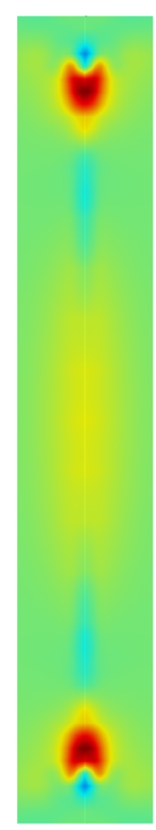

(c)

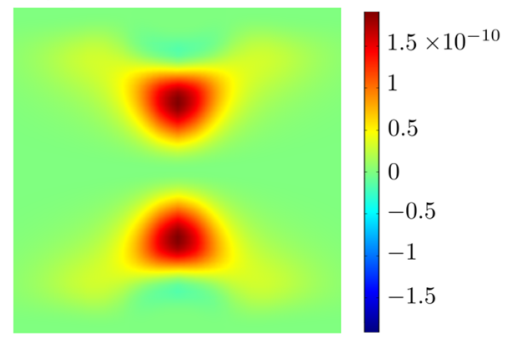

(f)

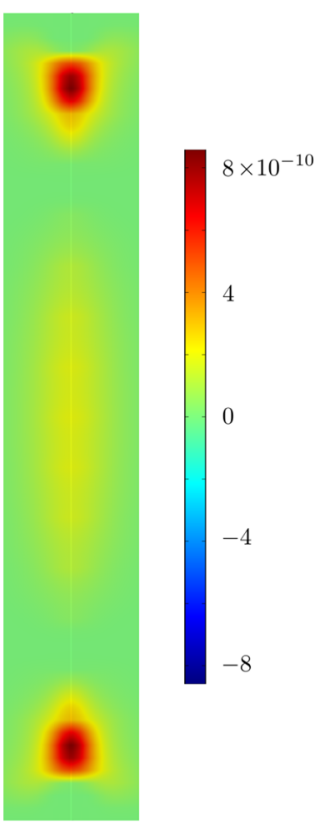

FIG. 18. Comparison between $S$ and $\dot{E}$ at $x=0$ for $A R=1[(\mathrm{a})-(\mathrm{c})]$ and $A R=6[(\mathrm{~d})-(\mathrm{f})] .[(\mathrm{a})$ and (d) $]$ Sensitivity $S,[(\mathrm{~b})$ and $(\mathrm{e})]$ time derivative $\dot{E}$ of total energy, and [(c) and (f)] component $\hat{v} \hat{v} \partial v_{0} / \partial y$ of $\dot{E}$. 
like in the present work. Interestingly, the minimum of $S$ is located at the center of the $\mathrm{X}$-junction where the vortex is generated.

For $A R=6$, Fig. 17(b), the sensitivity map displays one more lobe in the intersection of the channels than seen above for $A R=1$ ( 3 instead of 2). $S$ reaches its maximum values on the $z$-axis, but, here, there is a local maximum at $z=0$ instead of a minimum as for $A R=1$. The absolute maximum for $S$ occurs at two points close to the upper and lower walls for both $A R=6$ and 10 (in the latter case, $S$ displays four local maxima). This feature may be interesting for control applications using an external actuator because the most sensitive (target flow) region is close to the boundaries and not immersed in the bulk of the fluid. Interestingly, as $A R$ increases, the location of the maximum value for $S$ is closer to that of the maximum of $w_{0}$, although the latter is not involved in the definition of the sensitivity. On the other hand, for all $A R$ 's, the minimum of $S$ is located at the points where the vortices appear. Following Ref. 42, this suggests that in order to control the instability within $\mathrm{X}$-junctions, perturbations must not be applied at the centers of the vortices.

Despite the differences between the distributions of $S$ for different $A R$ 's, an important feature is that in all cases, the sensitivity $S$ is largest in the region of the interface where the two fluids first meet. For a T-shaped junction, Fani et al. ${ }^{40}$ also reported a maximum of the sensitivity in the crossing region. There are, indeed, two facing inlets in both the $\mathrm{X}$-junctions and $\mathrm{T}$-junctions; however, the values of $R e_{c}$ are lower in $\mathrm{X}$-junctions than in $\mathrm{T}$-junctions.

In order to understand better the instability, we compare now the spatial distributions of the sensitivity $S$ and of the time derivative of the kinetic energy per unit volume $\dot{E} \equiv \boldsymbol{u}^{\prime} \cdot \partial \boldsymbol{u}^{\prime} / \partial t$ for $A R$ $=1$, Figs. 18(a) $-18(\mathrm{c})$, and $A R=6$, Figs. 18(d) $-18(\mathrm{f})$. Larger values of $\dot{E}$ are concentrated in two $(A R=1)$ and three $(A R=6)$ main regions [Figs. 18(b) and 18(e)] with the maxima located very close to those of the sensitivity [Figs. 18(a) and 18(d)]. This emphasizes the importance of these regions for the development of the instability. Moreover, the dominant contribution to $\dot{E}$ corresponds to the exchange of energy between the perturbation and the base flow represented by the term $\hat{v} \hat{v} \partial v_{0} / \partial y,{ }^{38,52}$ as shown in Figs. 18(c) and $18(\mathrm{f})$; this is due to the strong $\hat{v}$-component and the large gradient of the $y$-component $v_{0}$ of the base flow, as shown in Sec. III B. This analysis of the energy exchange confirms, therefore, the important contribution of the interaction between the two facing flows in the inlets of the junction to the triggering of the instability.

\section{CONCLUSIONS}

In this paper, we have studied numerically the flow structure and the instabilities creating axial vortices in $\mathrm{X}$-junctions of perpendicular channels of rectangular cross sections with aspect ratios $A R$. Previous works had only dealt with either low values of $A R$ (one vortex ${ }^{23,26,49}$ or large ones (many vortices). ${ }^{53}$ The present study has been focused, instead, on a transition range: $1<A R<11$ for which $1-4$ vortices are observed.

We first used $3 D$ DNSs to determine the global structure of the flow field as a function of $A R$ and of the Reynolds number $R e=U_{\text {in }} \widetilde{W} / v$. Up to a critical Reynolds number $R e_{c}$, one has, for all aspect ratios, segregated outflows of the two fluids in the two outlets, each on a side of the mid-plane $y=0$. Above $R e_{c}$, steady vortex structures appear at the intersection of the junction and induce some local mixing of the fluids. Although the geometry of the domains is different, these steady vortex structures are reminiscent of the one reported by Kerr and Dold, who analyzed the stability of a stagnation point flow within an infinite domain.

For $1 \leq A R \leq 3.8$ and $R e \geq R e_{c}$, a single vortex with the axis parallel to each outlet develops, in good agreement with the results of Ref. 23. This feature has also been observed at the intersection of circular tubes in the range of crossing angles: $68^{\circ} \leq \alpha \leq 90^{\circ} . .^{24}$

For $A R \geq 3.8$, more vortices stacked along the $z$-axis appear for $R e>R e_{c}$ and their number $n$ increases steadily with $A R$. In the studied cases, the number of vortices only depends on $A R$ and not on $\operatorname{Re}\left(>R e_{c}\right)$. In addition, the instability leading to the appearance of the vortices is always subcritical irrespective of $A R$ : the vortices appear and disappear at different thresholds $R e_{c}$ (respectively, $R e_{c}^{*}$ ) when $R e$ follows an increasing (respectively, decreasing) ramp. $R e_{c}^{*}$ is always smaller than $R e_{c}$, and both numbers vary with $n$ but retain similar orders of magnitude.

Compared to the case $n=1$, the flow structures for $n=2,3$, and 4 display an important difference: for $n=1$, the vortex interlaces the streamlines of the two fluids across the whole section. For $n>1$, one observes instead, in the outlets, $n+1$ alternate stripes of the two pure fluids separated by zones close to the vortex centers where their streamlines are interlaced: mixing due to the vortices is, therefore, less thorough. Another important feature is that the normalized velocity profiles $v(z / H) / v_{\max , z}$ corresponding to different $A R$ values collapse precisely. As a result, the distance $d_{v}$ between adjacent vortices is proportional to $H$ and increases, therefore, with $A R$ for a given aperture $W$ : the velocity field corresponding to each vortex is then more and more elongated until a new vortex appears.

The DNSs are heavily time consuming, which makes difficult, for instance, the precise determination of the threshold of the instability. In order to obtain such information and understand better the dynamics of the system, we performed a global linear stability and sensitivity analysis in which the steady segregated flow is used as the base state. The critical values $R e_{c}$ for the transition from zero to $n$ vortices obtained in this way agree well with those determined from the DNS by increasing $R e$. For $R e>R e_{c}$, only one eigenvalue is both positive and real, which agrees with the idea that both zero and $n$ vortex configurations are steady, as observed in the DNSs. For a given $A R$ value, the corresponding eigenmode has the same number of vortices as that determined by the DNS, and the locations of these vortices predicted by both methods agree well.

The analysis of the leading eigenmodes also provides interesting information on the perturbation fields of the instability. They do not reach their highest values at the center of the intersection (origin of coordinates) but on the $z$-axis and close to the top and bottom walls (see Figs. 15 and 16). The sensitivity study, for which the adjoint modes must be considered, shows that in these spots, the receptivity to feedback local forces is highest: thus, these are the regions to actuate in order to control the instability. This result may be interpreted in terms of the kinetic energy variation with time, which reaches its maximum values close to the regions where $S$ is maximum; this variation is mostly due to the transfer of momentum from the base state to the $\hat{v}$ component (this agreement between the locations of the maxima of $S$ and the variation of the kinetic energy has been also reported for X-junctions but with three inlets and one outlet ${ }^{38}$ ). The sensitivity analysis also shows that the core of 
the instability is outside, and not inside, the vortex structures, as also occurring in T-junctions. ${ }^{36}$

As indicated above, the total computing time is significantly smaller for the global stability and sensitivity analysis than for the DNSs. Typically, the central processing unit (CPU) time to solve the non-linear problem is about eight times that required for the computation of five modes in the linear analysis (direct and adjoint problems). The linear analysis is, therefore, a robust alternative to study and predict the flow structure, and also for the detection of the most sensitive regions of the flow, which is a key asset for flow control strategies. However, computing the eigenmodes for large $A R$ values requires a large amount of memory for the meshing process. This has limited up to now the values of $A R$ that we have reached, and we are currently working to overcome this limitation.

We left the evaluation of the efficiency of active perturbations in the selection of one of the two steady flow configurations for future work. This control would be achieved by, for example, suction/injection of fluid through the upper and lower walls at the intersection. The technique should be appropriate for our geometry because the maximum of the sensitivity is measured close to the walls where it is easier to apply an active external perturbation. This control strategy was considered by Lashgari, ${ }^{38}$ also for X-junctions but with three inlets and one outlet, and it was found to be successful and might be applicable in the future work to our flow configuration. Another important issue to be considered in future works is the mixing efficiency of the junction, which is out of the scope of this article. As an illustration, we present a preliminary evaluation in the Appendix.

\section{ACKNOWLEDGMENTS}

This work was supported by public grants overseen by Secretaría de Políticas Universitarias through the program "Fortalecimiento de la Ciencia y Tecnología en las Universidades Nacionales" (Grant No. 03-PIO-44H) and by the French National Research Agency (ANR) through "Laboratoire d'Excellence Physics Atom Light Mater" (LabEx PALM) as part of the "Investissements d'Avenir" program (Grant No. ANR-10-LABX-0039-PALM). We also acknowledge support from Consejo Nacional de Investigaciones Científicas y Técnicas (CONICET) and the Franco-Argentinian International Research Project: "Ingénieries Vertes par la Mécanique des Fluides" (IRP-IVMF), CNRS-INSIS (France), and CONICET (Argentina). Data points from Ref. 23 have been included in Fig. 16 under the rules of the Creative Commons Attribution 3.0 License.

\section{APPENDIX: MIXING EFFICIENCY AND ASPECT RATIO}

As mentioned in the Introduction, junctions are potentially of interest for fluid mixing applications. ${ }^{55,56}$ We examined, therefore, briefly the mixing performance of the present $\mathrm{X}$-junctions by adding to our direct numerical simulations a transport equation and assuming a uniform concentration in each inlet (the dimensionless concentration is unity in one inlet and zero in the other). In a preliminary evaluation, we characterized mixing by the quality index $M^{47,55}$ defined by $M=1-\sqrt{\sigma^{2} / \sigma_{0}^{2}}$, where $\sigma^{2}$ is the variance of the concentration field in a section of the outlet channels and $\sigma_{0}^{2}$ corresponds to the maximum variance in this section. For a perfectly

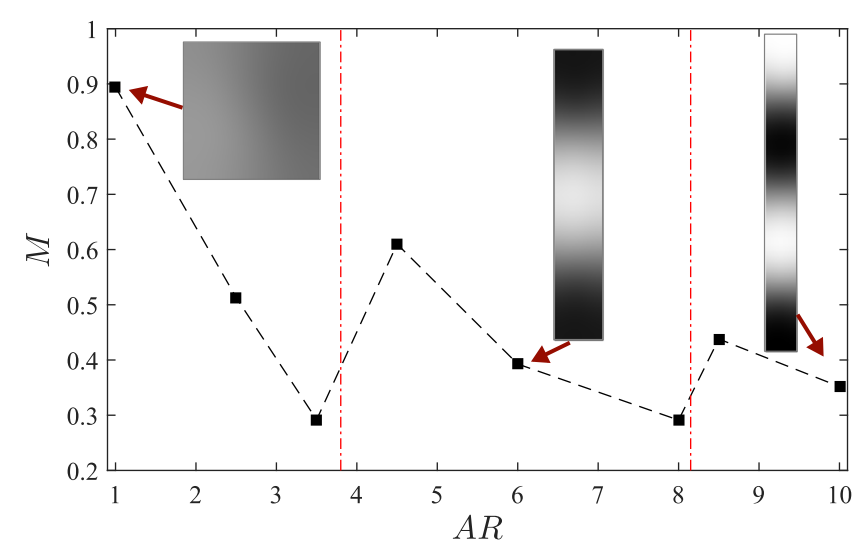

FIG. 19. Variation of the mixing quality index $M$ with the aspect ratio $A R$. Gray level images: maps of the dimensionless concentration at the cross section $x=7$, for $A R=1,6$, and 10 (arrows indicate $A R$ values for each map). Vertical dashed lines $=A R$ values corresponding to the transition between one and two vortices (left) and two and three vortices (right).

segregated flow, $M$ is equal to zero, and for a completely mixed one, $M=1$.

A qualitative trend of the mixing performance, for the range of $A R$ values considered here, is shown in Fig. 19: it displays the variation of $M$ with $A R$ at the cross section $x=7$ of one outlet channel, far from the center of the junctions. The index $M$ decreases with $A R$ for a fixed number $n$ of vortices: its value is then highest when $A R$ is near the lower limit of a range corresponding to a given $n$ value and lowest near the upper limit. This is likely due to the fact that the height along $z$ of a band of pure injected liquid located between two vortices (Fig. 2) is smaller near the lower limits mentioned above and larger near the upper ones. The local maximum of $M$ decreases with $n$ (mixing is most efficient for $A R=1$ in the range considered here), while the local minimum varies less. Summarizing, when a single junction is used, increasing $A R$ does not enhance mixing in itself but increases the number of alternate streams of the two fluids.

The development of new microfluidic and $3 D$ printing techniques opens the possibility to combine junctions ${ }^{57}$ and to build complex 3D structures with improved mixing characteristics. ${ }^{58,59}$ The formation of vortices and their localization in the network will be key factors of such improvements. In future studies, it will be interesting to consider as a first step the mixing properties of two junctions placed one behind the other.

\section{DATA AVAILABILITY}

The data that support the findings of this study are available within this article.

\section{REFERENCES}

${ }^{1}$ L. Capretto, W. Cheng, M. Hill, and X. Zhang, "Micromixing within microfluidic devices," in Microfluidics, Topics in Current Chemistry Vol. 304, edited by J. Fagerberg, D. C. Mowery, and R. R. Nelson (Springer, Berlin, Heidelberg, 2011). ${ }^{2}$ C.-Y. Lee, W.-T. Wang, C.-C. Liu, and L.-M. Fu, "Passive mixers in microfluidic systems: A review,” Chem. Eng. J. 288, 146-160 (2016). 
${ }^{3}$ D. R. Gossett, W. M. Weaver, A. J. Mach, S. C. Hur, H. T. K. Tse, W. Lee, H. Amini, and D. Di Carlo, "Label-free cell separation and sorting in microfluidic systems," Anal. Bioanal. Chem. 397, 3249-3267 (2010).

${ }^{4}$ E. L. Jackson and H. Lu, "Advances in microfluidic cell separation and manipulation,” Curr. Opin. Chem. Eng. 2, 398-404 (2013).

${ }^{5}$ P. Sajeesh and A. K. Sen, "Particle separation and sorting in microfluidic devices: A review," Microfluid. Nanofluid. 17, 1-52 (2014).

${ }^{6}$ J. Nilsson, M. Evander, B. Hammarström, and T. Laurell, "Review of cell and particle trapping in microfluidic systems," Anal. Chim. Acta 649, 141-157 (2009).

${ }^{7}$ H. Amini, E. Sollier, W. M. Weaver, and D. Di Carlo, "Intrinsic particle-induced lateral transport in microchannels," Proc. Natl. Acad. Sci. U. S. A. 109, 1159311598 (2012).

${ }^{8}$ W. M. Abed, A. F. Domingues, R. J. Poole, and D. J. C. Dennis, "Heat transfer enhancement in a cross-slot micro-geometry," Int. J. Therm. Sci. 121, 249-265 (2017).

${ }^{9}$ P. B. Howell, Jr., D. R. Mott, S. Fertig, C. R. Kaplan, J. P. Golden, E. S. Oran, and F. S. Ligler, "A microfluidic mixer with grooves placed on the top and bottom of the channel," Lab Chip 5, 524-530 (2005).

${ }^{10}$ A. P. Sudarsan and V. M. Ugaz, "Multivortex micromixing," Proc. Natl. Acad. Sci. U. S. A. 103, 7228-7233 (2006).

${ }^{11}$ A. P. Sudarsan and V. M. Ugaz, "Fluid mixing in planar spiral microchannels," Lab Chip 6, 74-82 (2006).

${ }^{12}$ V. Kumar, M. Paraschivoiu, and K. D. P. Nigam, "Single-phase fluid flow and mixing in microchannels," Chem. Eng. Sci. 66, 1329-1373 (2011).

${ }^{13}$ Y. A. Aryshev, V. A. Golovin, and S. A. Ershin, "Stability of colliding flows," Fluid Dyn. 16, 755-759 (1981).

${ }^{14}$ S. J. Haward, G. H. McKinley, and A. Q. Shen, "Elastic instabilities in planar elongational flow of monodisperse polymer solutions," Sci. Rep. 6, 33029 (2016).

${ }^{15}$ T. T. Perkins, D. E. Smith, and S. Chu, "Single polymer dynamics in an elongational flow," Science 276, 2016-2021 (1997).

${ }^{16} \mathrm{~W}$. Xu and S. J. Muller, "Exploring both sequence detection and restriction endonuclease cleavage kinetics by recognition site via single-molecule microfluidic trapping," Lab Chip 11, 435-442 (2011).

${ }^{17}$ G. N. Rocha, R. J. Poole, M. A. Alves, and P. J. Oliveira, "On extensibility effects in the cross-slot flow bifurcation," J. Non-Newtonian Fluid Mech. 156, 58-69 (2009).

${ }^{18}$ S. J. Haward and G. H. McKinley, "Stagnation point flow of wormlike micellar solutions in a microfluidic cross-slot device: Effects of surfactant concentration and ionic environment," Phys. Rev. E 85, 031502 (2012).

${ }^{19}$ P. E. Arratia, C. C. Thomas, J. Diorio, and J. P. Gollub, "Elastic instabilities of polymer solutions in cross-channel flow," Phys. Rev. Lett. 96, 144502 (2006).

${ }^{20}$ S. J. Haward, "Microfluidic extensional rheometry using stagnation point flow," Biomicrofluidics 10, 043401 (2016).

${ }^{21}$ N. Burshtein, K. Zografos, A. Q. Shen, R. Poole, and S. J. Haward, "Inertioelastic flow instability at a stagnation point," Phys. Rev. X 7, 041039 (2017).

${ }^{22} \mathrm{~V}$. Kantsler, E. Segre, and V. Steinberg, "Critical dynamics of vesicle stretching transition in elongational flow," Phys. Rev. Lett. 101, 048101 (2008).

${ }^{23}$ S. J. Haward, R. J. Poole, M. A. Alves, P. J. Oliveira, N. Goldenfeld, and A. Q. Shen, "Tricritical spiral vortex instability in cross-slot flow," Phys. Rev. E 93, 031101 (2016)

${ }^{24}$ P. G. Correa, J. R. Mac Intyre, J. M. Gomba, M. A. Cachile, J. P. Hulin, and H. Auradou, "Three-dimensional flow structures in X-shaped junctions: Effect of the Reynolds number and crossing angle," Phys. Fluids 31, 043606 (2019).

${ }^{25}$ W. R. Dean, "LXXII. The stream-line motion of fluid in a curved pipe (Second paper)," Philos. Mag. 5, 673 (1928).

${ }^{26}$ J. W. Zhang, W. F. Li, X. L. Xu, H. F. Liu, and F. C. Wang, "Experimental investigation of three-dimensional flow regimes in a cross-shaped reactor," Phys. Fluids 31, 034105 (2019)

${ }^{27}$ N. Burshtein, A. Q. Shen, and S. J. Haward, "Controlled symmetry breaking and vortex dynamics in intersecting flows," Phys. Fluids 31, 034104 (2019).

${ }^{28}$ G. Y. Kang, D. W. Carlson, T. H. Kang, S. Lee, S. J. Haward, I. Choi, A. Q. Shen, and A. J. Chung, "Intracellular nanomaterial delivery via spiral hydroporation," ACS Nano 14, 3048-3058 (2020).
${ }^{29}$ I. Damian, S. Hardt, and C. Balan, "From flow focusing to vortex formation in crossing microchannels," Microfluid. Nanofluid. 21, 142 (2017).

${ }^{30}$ A. Borgogna, M. Murmura, M. Annesini, M. Giona, and S. Cerbelli, "Inertiadriven enhancement of mixing efficiency in microfluidic cross-junctions: A combined eulerian/Lagrangian approach," Microfluid. Nanofluid. 22, 20 (2018).

${ }^{31}$ R. Moloudi, S. Oh, C. Yang, M. E. Warkiani, and M. W. Naing, "Inertial particle focusing dynamics in a trapezoidal straight microchannel: Application to particle filtration," Microfluid. Nanofluid. 22, 33 (2018).

${ }^{32}$ S. H. Lee and P. K. Kang, "Three-dimensional vortex-induced reaction hot spots at flow intersections," Phys. Rev. Lett. 124, 144501 (2020).

${ }^{33}$ S. T. Chan, S. J. Haward, and A. Q. Shen, "Microscopic investigation of vortex breakdown in a dividing T-junction flow," Phys. Rev. Fluids 3, 072201(R) (2018).

${ }^{34}$ V. N. Kalashnikov and M. G. Tsiklauri, "Ordered three-dimensional structures resulting from instability of two-dimensional flow in crossed channels," Fluid Dyn. 26, 161-165 (1991).

${ }^{35}$ F. Giannetti and P. Luchini, "Structural sensitivity of the first instability of the cylinder wake,” J. Fluid Mech. 581, 167-197 (2007).

${ }^{36} \mathrm{~K}$. K. Chen, C. W. Rowley, and H. A. Stone, "Vortex breakdown, linear global instability and sensitivity of pipe bifurcation flows," J. Fluid Mech. 815, 257-294 (2017).

${ }^{37}$ O. Marquet, D. Sipp, and L. Jacquin, "Sensitivity analysis and passive control of cylinder flow," J. Fluid Mech. 615, 221-252 (2008).

${ }^{38}$ I. Lashgari, O. Tammisola, V. Citro, M. P. Juniper, and L. Brandt, "The planar X-junction flow: Stability analysis and control," J. Fluid Mech. 753, 1-28 (2014).

${ }^{39}$ V. Theofilis, "Global linear instability," Annu. Rev. Fluid Mech. 43, 319-352 (2011).

${ }^{40}$ A. Fani, S. Camarri, and M. V. Salvetti, "Investigation of the steady engulfment regime in a three-dimensional T-mixer," Phys. Fluids 25, 064102 (2013).

${ }^{41}$ M. Cachile, L. Talon, J. M. Gomba, J. P. Hulin, and H. Auradou, "Stokes flow paths separation and recirculation cells in X-junctions of varying angle," Phys. Fluids 24, 021704 (2012).

${ }^{42} \mathrm{~J}$.-M. Chomaz, "Global instabilities in spatially developing flows: Non-normality and nonlinearity," Annu. Rev. Fluid Mech. 37, 357-392 (2005).

${ }^{43} \mathrm{D}$. Hill, "A theoretical approach for analyzing the restabilization of wakes," in 30th Aerospace Sciences Meeting and Exhibit (AIAA, 1992), p. 920067.

${ }^{44} \mathrm{M}$. P. Juniper and B. Pier, "The structural sensitivity of open shear flows calculated with a local stability analysis," Eur. J. Mech., B: Fluids 49, 426-437 (2015).

${ }^{45}$ U. A. Qadri, D. Mistry, and M. P. Juniper, "Structural sensitivity of spiral vortex breakdown," J. Fluid Mech. 720, 558-581 (2013).

${ }^{46}$ R. B. Lehoucq, D. C. Sorensen, and C. Yang, ARPACK Users' Guide (SIAM, 1998).

${ }^{47}$ M. Engler, N. Kockmann, T. Kiefer, and P. Woias, "Numerical and experimental investigations on liquid mixing in static micromixers," Chem. Eng. J. 101, 315-322 (2004).

${ }^{48}$ A. Soleymani, E. Kolehmainen, and I. Turunen, "Numerical and experimental investigations of liquid mixing in T-type micromixers," Chem. Eng. J. 135, S219S228 (2008).

${ }^{49}$ J. W. Zhang, T. L. Yao, W. F. Li, M. El Hassan, X. L. Xu, H. F. Liu, and F. C. Wang, "Trapping region of impinging jets in a cross-shaped channel," AIChE J. 66, e16822 (2020).

${ }^{50} \mathrm{~J}$. Jeong and F. Hussain, "On the identification of a vortex," J. Fluid Mech. 285, 69-94 (1995).

${ }^{51}$ P. Chakraborty, S. Balachandar, and R. J. Adrian, "On the relationships between local vortex identification schemes," J. Fluid Mech. 535, 189-214 (2005).

${ }^{52} \mathrm{P}$. Schmid and D. Henningson, Stability and Transition in Shear Flows, Applied Mathematical Sciences Vol. 142 (Springer-Verlag, New York, 2001).

${ }^{53}$ V. N. Kalashnikov and M. G. Tsiklauri, "Effect of polymer additives on ordered three-dimensional structures arising in cross-slot flow," J. Non-Newtonian Fluid Mech. 48, 215-223 (1993).

${ }^{54}$ O. S. Kerr and J. W. Dold, "Periodic steady vortices in a stagnation-point flow," J. Fluid Mech. 276, 307-325 (1994). 
${ }^{55}$ S. Dreher, N. Kockmann, and P. Woias, "Characterization of laminar transient flow regimes and mixing in T-shaped micromixers," Heat Transfer Eng. 30, 91100 (2009).

${ }^{56}$ A. S. Lobasov and A. V. Minakov, "Analyzing mixing quality in a T-shaped micromixer for different fluids properties through numerical simulation," Chem. Eng. Process. 124, 11-23 (2018).
${ }^{57}$ S. J. Haward, C. C. Hopkins, K. Toda-Peters, and A. Q. Shen, "Microfluidic analog of an opposed-jets device," Appl. Phys. Lett. 114, 223701 (2019).

${ }^{58} \mathrm{~K}$. C. Bhargava, B. Thompson, and N. Malmstadt, "Discrete elements for 3D microfluidics," Proc. Natl. Acad. Sci. U. S. A. 111, 15013-15018 (2014).

${ }^{59}$ X. Lai, Z. Shi, Z. Pu, P. Zhang, X. Zhang, H. Yu, and D. Li, "A Rubik's microfluidic cube," Microsyst. Nanoeng. 6, 27 (2020). 